\title{
-Supplementary Material- Investigating the (poly)radicaloid nature of real-world organic compounds by DFT-based methods
}

\author{
G. Salvitti ${ }^{a, b}$, F. Negri ${ }^{b, c}$, \\ A.J. Pérez-Jiménez ${ }^{a}$, E. San-Fabián ${ }^{a}$, \\ D. Casanova ${ }^{d, e}$, and J. C. Sancho-García ${ }^{a *}$ \\ ${ }^{a}$ Department of Physical Chemistry, \\ University of Alicante, \\ E-03080 Alicante, Spain \\ ${ }^{b}$ Dipartimento di Chimica "Giacomo Ciamician", \\ Università di Bologna, \\ IT-40126 Bologna, Italy \\ ${ }^{c}$ INSTM UdR Bologna, Italy \\ ${ }^{d}$ Donostia International Physics Center (DIPC), \\ E-20018 Donostia, Spain \\ ${ }^{e}$ IKERBASQUE, \\ Basque Foundation for Science, \\ E-48013 Bilbao, Spain
}

${ }^{*}$ E-mail: jc.sancho@ua.es 


\section{Contents}

1 Error estimates $\quad 3$

2 Results from the RAS-SF method 3

3 Radical(oid) indices $\quad 4$

4 Calculation of ZFS tensor $\quad 5$

4.1 Theoretical estimates of the exciton size . . . . . . 8

5 Cartesian coordinates of all compounds $\quad 8$ 


\section{Error estimates}

When needed, the Mean Signed Error (MSE) and Mean Unsigned Error (MUE) will be used to estimate the deviations between computational $\left(a_{i}\right)$ and experimental $\left(e_{i}\right)$ results for energy differences:

$$
\begin{aligned}
\mathrm{MSE} & =\frac{1}{N} \sum_{i}^{N}\left(a_{i}-e_{i}\right), \\
\mathrm{MUE} & =\frac{1}{N} \sum_{i}^{N}\left|a_{i}-e_{i}\right|,
\end{aligned}
$$

as well as the Minimum (MIN) and Maximum (MAX) deviations.

\section{Results from the RAS-SF method}

Table S1: $N_{U}$ and energy difference (kcal/mol) between the low-spin (LS) and high-spin (HS) states, $\Delta E(\mathrm{LS}-\mathrm{HS})$, obtained at the RAS-SF level.

\begin{tabular}{lcrrrr}
\hline Compound & GS & $N_{U}(\mathrm{LS})$ & $N_{U}(\mathrm{HS})$ & $\Delta E(\mathrm{LS}-\mathrm{HS})$ & Exp. \\
\hline Diradical I & $\mathrm{T}_{0}$ & 2.03 & 2.08 & $0.28^{\mathrm{a}}$ & $0.50 \pm 0.02$ \\
Diradical II & $\mathrm{T}_{0}$ & 2.05 & 2.09 & $1.12^{\mathrm{a}}$ & $1.74 \pm 0.07$ \\
[6]CPPM-Mes & $\mathrm{S}_{0}$ & 2.54 & 3.43 & $-9.41^{\mathrm{b}}$ & $-6.23 \pm 0.78$ \\
[3]CPBM-Ant & $\mathrm{D}_{0}$ & 1.70 & 3.00 & $-4.19^{\mathrm{c}}$ & -3.5 \\
[4]CPBM-Ant & $\mathrm{S}_{0}$ & 2.95 & 3.47 & $-3.40^{\mathrm{c}}$ & -2.8 \\
[5]CPBM-Ant & $\mathrm{D}_{0}$ & 3.94 & 4.51 & $-3.40^{\mathrm{c}}$ & -2.4 \\
[6]CPBM-Ant & $\mathrm{S}_{0}$ & 4.61 & 4.98 & $-1.63^{\mathrm{c}}$ & -2.2 \\
MC-F3A3 & $\mathrm{D}_{0}$ & 2.58 & 3.00 & $-1.66^{\mathrm{a}}$ & -2.10 \\
8MC & $\mathrm{S}_{0}$ & 4.85 & 5.39 & $-2.70^{\mathrm{d}}$ & -3.08 \\
\hline
\end{tabular}

${ }^{a}$ Done here (see Computational Details).

b Taken from Wu et al. ${ }^{1}$

c Taken from $\mathrm{Wu}$ et al. ${ }^{2}$

d Taken from Wu et al. ${ }^{3}$ 


\section{Radical(oid) indices}

Table S2: Calculated radical indices $y_{i}^{\alpha}$ by the FT-DFT method at the TPSS0/def2TZVP level.

\begin{tabular}{lcccc} 
Compound & $y_{0}^{\alpha}$ & $y_{1}^{\alpha}$ & $y_{2}^{\alpha}$ & $y_{3}^{\alpha}$ \\
\hline Diradical I & 0.49 & 0.06 & 0.05 & 0.02 \\
Diradical II & 0.49 & 0.07 & 0.02 & 0.02 \\
[6]CPPM-Mes & 0.31 & 0.29 & 0.10 & 0.03 \\
[3]CPBM-Ant & 0.37 & 0.13 & 0.13 & 0.13 \\
[4]CPBM-Ant & 0.32 & 0.27 & 0.11 & 0.11 \\
[5]CPBM-Ant & 0.35 & 0.33 & 0.12 & 0.12 \\
[6]CPBM-Ant & 0.37 & 0.33 & 0.27 & 0.11 \\
MC-F3A3 & 0.52 & 0.12 & 0.12 & 0.12 \\
8MC & 0.37 & 0.37 & 0.30 & 0.30 \\
\hline
\end{tabular}

Table S3: Calculated radical indices $y_{i}^{\alpha}$ by the FT-DFT method at the TPSSHH/def2TZVP level.

\begin{tabular}{lcccc} 
Compound & $y_{0}^{\alpha}$ & $y_{1}^{\alpha}$ & $y_{2}^{\alpha}$ & $y_{3}^{\alpha}$ \\
\hline Diradical I & 0.48 & 0.08 & 0.07 & 0.03 \\
Diradical II & 0.49 & 0.09 & 0.04 & 0.03 \\
[6]CPPM-Mes & 0.32 & 0.31 & 0.13 & 0.05 \\
[3]CPBM-Ant & 0.36 & 0.15 & 0.15 & 0.15 \\
[4]CPBM-Ant & 0.33 & 0.29 & 0.14 & 0.14 \\
[5]CPBM-Ant & 0.36 & 0.34 & 0.15 & 0.15 \\
[6]CPBM-Ant & 0.38 & 0.34 & 0.29 & 0.14 \\
MC-F3A3 & 0.53 & 0.14 & 0.14 & 0.14 \\
8MC & 0.40 & 0.39 & 0.33 & 0.33 \\
\hline
\end{tabular}




\section{Calculation of ZFS tensor}

By default, the g-tensor is used as reference tensor for $|D|<0.3 \mathrm{~cm}^{-1}$, with its axes assigned depending on their magnitude:

$$
\begin{aligned}
\left|g_{\min }\right\rangle & \longrightarrow\left|g_{x}\right\rangle, \\
\left|g_{\text {mid }}\right\rangle & \longrightarrow\left|g_{y}\right\rangle, \\
\left|g_{\max }\right\rangle & \longrightarrow\left|g_{z}\right\rangle,
\end{aligned}
$$

and thus proper care should be given if previous or future calculations reassign this ordering. The D-tensor is used as reference only if $S>1 / 2$ and $|D|>0.3 \mathrm{~cm}^{-1}$, which is not the case here. 
Table S4: Comparison between calculated and experimental EPR parameter for the triplet state of Diradical I and II, with the EPR-II basis set.

\begin{tabular}{lrrrrrr} 
& \multicolumn{3}{c}{ Diradical I } & \multicolumn{3}{c}{ Diradical II } \\
\cline { 2 - 7 } Method & $\mathrm{D} / \mathrm{hc}\left(10^{3} \mathrm{~cm}^{-1}\right)$ & $\mathrm{E} / \mathrm{hc}\left(10^{3} \mathrm{~cm}^{-1}\right)$ & $\mathrm{g}$-tensor & $\mathrm{D} / \mathrm{hc}\left(10^{3} \mathrm{~cm}^{-1}\right)$ & $\mathrm{E} / \mathrm{hc}\left(10^{3} \mathrm{~cm}^{-1}\right)$ & $\mathrm{g}$-tensor \\
\hline HF & -5.03 & -0.94 & 2.0063 & 12.37 & 2.13 & 2.0064 \\
HF-PBE & -5.11 & -0.93 & 2.0064 & 12.32 & 2.27 & 2.0065 \\
TPSS & -5.42 & -1.68 & 2.0048 & 12.14 & 3.30 & 2.0048 \\
TPSS0 & -5.42 & -1.28 & 2.0052 & 11.87 & 3.25 & 2.0052 \\
TPSSHH & -5.39 & -1.10 & 2.0056 & 11.77 & 3.01 & 2.0056 \\
PBE0 & -5.44 & -1.28 & 2.0053 & 11.90 & 3.34 & 2.0053 \\
M06-2X & -5.53 & -1.02 & 2.0064 & 11.75 & 3.58 & 2.0064 \\
CAM-B3LYP & -5.51 & -0.96 & 2.0054 & 11.58 & 3.57 & 2.0055 \\
LC-BLYP & -5.58 & -0.80 & 2.0054 & -11.48 & -3.72 & 2.0054 \\
$\omega$ B97XD & -5.56 & -0.83 & 2.0055 & 11.41 & 3.80 & 2.0055 \\
Exp. values & & -0.14 & 2.0044 & -8.08 & -1.17 & 2.0050 \\
\hline a Taken & -2.32 & & & &
\end{tabular}

a Taken from Rajca et al. ${ }^{4}$ 
Table S5: Comparison between calculated and experimental EPR parameter for the triplet state of Diradical I and II, with the IGLO-II basis set.

\begin{tabular}{|c|c|c|c|c|c|c|}
\hline \multirow[b]{2}{*}{ Method } & \multicolumn{3}{|c|}{ Diradical I } & \multicolumn{3}{|c|}{ Diradical II } \\
\hline & $\mathrm{D} / \mathrm{hc}\left(10^{3} \mathrm{~cm}^{-1}\right)$ & $\mathrm{E} / \mathrm{hc}\left(10^{3} \mathrm{~cm}^{-1}\right)$ & g-tensor & $\mathrm{D} / \mathrm{hc}\left(10^{3} \mathrm{~cm}^{-1}\right)$ & $\mathrm{E} / \mathrm{hc}\left(10^{3} \mathrm{~cm}^{-1}\right)$ & g-tensor \\
\hline TPSS & -5.37 & -1.60 & 2.0049 & 11.81 & 3.26 & 2.0049 \\
\hline TPSS0 & -5.37 & -1.23 & 2.0053 & 11.49 & 3.21 & 2.0053 \\
\hline TPSSHH & -5.35 & -1.06 & 2.0057 & 11.34 & 2.97 & 2.0057 \\
\hline PBE0 & -5.39 & -1.22 & 2.0055 & 11.53 & 3.28 & 2.0055 \\
\hline M06-2X & -5.48 & -0.98 & 2.0065 & 11.35 & 3.52 & 2.0066 \\
\hline CAM-B3LYP & -5.47 & -0.92 & 2.0056 & 11.22 & 3.51 & 2.0056 \\
\hline LC-BLYP & -5.54 & -0.77 & 2.0056 & -11.20 & -3.59 & 2.0056 \\
\hline$\omega \mathrm{B} 97 \mathrm{XD}$ & -5.52 & -0.80 & 2.0056 & -11.13 & -3.66 & 2.0056 \\
\hline Exp. values ${ }^{\mathrm{a}}$ & -2.32 & -0.14 & 2.0044 & -8.08 & -1.17 & 2.0050 \\
\hline
\end{tabular}

a Taken from Rajca et al. ${ }^{4}$ 


\subsection{Theoretical estimates of the exciton size}

If the spin-orbit and crystal-field contributions to the ZFS are neglected, which is a reasonable assumption for organic systems, ${ }^{5}$ the $D$ parameter can be formulated as: ${ }^{6}$

$$
D=\frac{3}{4} \frac{\mu_{0}}{4 \pi h}\left(g_{e} \mu_{B}\right)^{2}\left\langle\frac{1-3 \cos ^{2} \theta}{r^{3}}\right\rangle,
$$

with $r$ the distance between the spins of the unpaired electrons, $\theta$ the angle between the spin vectors and the dipolar $z$-axis, and $\mu_{0}, g_{e}$, and $\mu_{B}$ physical constants. Note that the sign of $D$ depends on the $\left(1-3 \cos ^{2} \theta\right)$ term, with $D>0(D<0)$ an oblate (prolate) shape of the spin distribution. If $D$ is given in $\mathrm{cm}^{-1}$, while using $\AA$ as length unit, one arrives to the expression:

$$
|D|=0.6495 \frac{g^{2}}{r_{A B}^{3}},
$$

from which we estimate the exciton size $r_{A B}$ (or simply $\Delta r$ ) as that determining:

$$
|D| \propto \iint \sigma_{A}\left(\mathbf{r}_{A}\right) \frac{1}{r_{A B}^{3}} \sigma_{B}\left(\mathbf{r}_{B}\right) d \mathbf{r}_{A} d \mathbf{r}_{B},
$$

in terms of spin densities around radical centers $A$ and $B$.

\section{Cartesian coordinates of all compounds}

All the calculations were performed at the molecular geometries extracted from literature, to consistenly compare FT-DFT, SF-DFT, SF-TDDFT, and RAS-SF methods. The exception was the MC-F3A3 system, for which the B97-3c composite method ${ }^{7}$ was used here. 


\begin{tabular}{|c|c|c|c|}
\hline \multicolumn{4}{|c|}{ Diradical-I } \\
\hline $\mathrm{C}$ & 0.00981 & -0.05729 & -0.00718 \\
\hline $\mathrm{C}$ & 0.00284 & -0.06449 & 1.37993 \\
\hline $\mathrm{C}$ & 1.20113 & -0.04103 & 2.11492 \\
\hline $\mathrm{C}$ & 2.42243 & 0.02925 & 1.38568 \\
\hline $\mathrm{C}$ & 2.41616 & -0.00199 & -0.01325 \\
\hline $\mathrm{C}$ & 1.21595 & -0.04157 & -0.73476 \\
\hline $\mathrm{H}$ & -0.93886 & -0.07868 & -0.52936 \\
\hline $\mathrm{H}$ & -0.92556 & -0.10596 & 1.93970 \\
\hline $\mathrm{H}$ & 3.36039 & -0.00232 & -0.54187 \\
\hline $\mathrm{C}$ & 2.36047 & -0.18945 & 4.09043 \\
\hline $\mathrm{N}$ & 1.17170 & -0.11628 & 3.48074 \\
\hline $\mathrm{N}$ & 3.56451 & -0.10235 & 3.51278 \\
\hline $\mathrm{N}$ & 3.58599 & 0.08809 & 2.16158 \\
\hline $\mathrm{C}$ & 2.37179 & -0.37006 & 5.56780 \\
\hline $\mathrm{C}$ & 3.57561 & -0.39752 & 6.28888 \\
\hline $\mathrm{C}$ & 1.15934 & -0.51625 & 6.25773 \\
\hline $\mathrm{C}$ & 3.56361 & -0.57044 & 7.67093 \\
\hline $\mathrm{H}$ & 4.51196 & -0.27827 & 5.75644 \\
\hline $\mathrm{C}$ & 1.15210 & -0.68777 & 7.64038 \\
\hline $\mathrm{H}$ & 0.23514 & -0.49174 & 5.69237 \\
\hline $\mathrm{C}$ & 2.35296 & -0.71629 & 8.35207 \\
\hline $\mathrm{H}$ & 4.50165 & -0.58918 & 8.21842 \\
\hline $\mathrm{H}$ & 0.20667 & -0.80006 & 8.16343 \\
\hline $\mathrm{H}$ & 2.34609 & -0.85018 & 9.43010 \\
\hline $\mathrm{C}$ & 4.89219 & 0.29299 & 1.62749 \\
\hline $\mathrm{C}$ & 5.94886 & -0.51306 & 2.06360 \\
\hline $\mathrm{C}$ & 5.14314 & 1.32426 & 0.71516 \\
\hline $\mathrm{C}$ & 7.23267 & -0.31329 & 1.57425 \\
\hline $\mathrm{H}$ & 5.75301 & -1.29318 & 2.78934 \\
\hline $\mathrm{C}$ & 6.42577 & 1.52984 & 0.22225 \\
\hline $\mathrm{H}$ & 4.33926 & 1.98328 & 0.40716 \\
\hline $\mathrm{C}$ & 7.49693 & 0.70921 & 0.63644 \\
\hline $\mathrm{H}$ & 8.04153 & -0.95039 & 1.90184 \\
\hline $\mathrm{H}$ & 6.61616 & 2.33724 & -0.47003 \\
\hline $\mathrm{C}$ & 8.84697 & 0.91843 & 0.11301 \\
\hline $\mathrm{C}$ & 10.63957 & 1.72245 & -1.23565 \\
\hline $\mathrm{C}$ & 11.21012 & 0.75909 & -0.15065 \\
\hline $\mathrm{N}$ & 9.23265 & 1.92103 & -0.72788 \\
\hline $\mathrm{N}$ & 9.93828 & 0.14400 & 0.37980 \\
\hline $\mathrm{O}$ & 10.00109 & -0.89755 & 1.11435 \\
\hline $\mathrm{O}$ & 8.51489 & 2.85827 & -1.21375 \\
\hline $\mathrm{C}$ & 11.86582 & 1.47190 & 1.04612 \\
\hline $\mathrm{H}$ & 12.03394 & 0.73688 & 1.83660 \\
\hline $\mathrm{H}$ & 12.82701 & 1.90778 & 0.76164 \\
\hline $\mathrm{H}$ & 11.22702 & 2.26425 & 1.44492 \\
\hline $\mathrm{C}$ & 12.13090 & -0.34686 & -0.66545 \\
\hline $\mathrm{H}$ & 13.05569 & 0.08974 & -1.05377 \\
\hline $\mathrm{H}$ & 12.37859 & -1.01837 & 0.15797 \\
\hline $\mathrm{H}$ & 11.66561 & -0.94013 & -1.45367 \\
\hline $\mathrm{C}$ & 11.33315 & 3.07969 & -1.34795 \\
\hline $\mathrm{H}$ & 12.35633 & 2.94591 & -1.71118 \\
\hline $\mathrm{H}$ & 10.78729 & 3.70234 & -2.05829 \\
\hline $\mathrm{H}$ & 11.36616 & 3.60880 & -0.39472 \\
\hline $\mathrm{C}$ & 10.49862 & 1.09147 & -2.63301 \\
\hline $\mathrm{H}$ & 9.89829 & 1.76021 & -3.25416 \\
\hline $\mathrm{H}$ & 11.47676 & 0.95998 & -3.10264 \\
\hline $\mathrm{H}$ & 9.99801 & 0.12053 & -2.59167 \\
\hline $\mathrm{C}$ & 1.26185 & -0.07399 & -2.27387 \\
\hline $\mathrm{C}$ & 1.99309 & 1.18532 & -2.79723 \\
\hline $\mathrm{H}$ & 3.02181 & 1.24293 & -2.42969 \\
\hline $\mathrm{H}$ & 2.03278 & 1.17454 & -3.89214 \\
\hline $\mathrm{H}$ & 1.47412 & 2.09767 & -2.48584 \\
\hline $\mathrm{C}$ & 2.02282 & -1.33853 & -2.73946 \\
\hline $\mathrm{H}$ & 3.05065 & -1.35752 & -2.36552 \\
\hline $\mathrm{H}$ & 1.52317 & -2.24739 & -2.38912 \\
\hline $\mathrm{H}$ & 2.06643 & -1.37461 & -3.83370 \\
\hline $\mathrm{C}$ & -0.14564 & -0.10414 & -2.89948 \\
\hline $\mathrm{H}$ & -0.70852 & -0.99290 & -2.59651 \\
\hline $\mathrm{H}$ & -0.73146 & 0.78101 & -2.63096 \\
\hline $\mathrm{H}$ & -0.06077 & -0.12420 & -3.99052 \\
\hline
\end{tabular}




\begin{tabular}{crrr} 
Table & \multicolumn{1}{c}{ S4: } & Cartesian & coordinates \\
Diradical-II & & & \\
\hline $\mathrm{H}$ & 7.33160 & 0.14670 & 7.55590 \\
$\mathrm{H}$ & 6.75050 & 1.58910 & 5.61270 \\
$\mathrm{C}$ & 6.32920 & 0.12250 & 7.13850 \\
$\mathrm{C}$ & 6.00320 & 0.93070 & 6.04650 \\
$\mathrm{H}$ & 5.60640 & -1.35930 & 8.53020 \\
$\mathrm{C}$ & 5.36180 & -0.71900 & 7.68810 \\
$\mathrm{C}$ & 4.71830 & 0.91120 & 5.51260 \\
$\mathrm{H}$ & 4.43510 & 1.55160 & 4.68710 \\
$\mathrm{C}$ & 4.07350 & -0.75520 & 7.15770 \\
$\mathrm{H}$ & 2.66250 & 0.40290 & 12.78170 \\
$\mathrm{C}$ & 3.75080 & 0.07570 & 6.08080 \\
$\mathrm{H}$ & 3.33090 & -1.42220 & 7.57840 \\
$\mathrm{C}$ & 1.71180 & 0.92950 & 12.88380 \\
$\mathrm{O}$ & 2.26000 & -0.07550 & 10.27990 \\
$\mathrm{H}$ & 1.41730 & 0.92760 & 13.93810 \\
$\mathrm{H}$ & 3.42750 & -0.00780 & 1.94920 \\
$\mathrm{H}$ & 2.15210 & 0.34370 & 8.20710 \\
$\mathrm{H}$ & 1.57060 & -1.71470 & 12.26120 \\
$\mathrm{~N}$ & 2.44350 & 0.06340 & 5.51080 \\
$\mathrm{H}$ & 3.48040 & -0.07330 & -0.53400 \\
$\mathrm{~N}$ & 2.43240 & 0.07560 & 4.15290 \\
$\mathrm{~N}$ & 1.06430 & 0.23870 & 10.60120 \\
$\mathrm{C}$ & 0.65390 & 0.21590 & 12.04720 \\
$\mathrm{C}$ & 0.58960 & -1.26790 & 12.44100 \\
$\mathrm{H}$ & 0.33710 & -1.38110 & 13.49910 \\
$\mathrm{C}$ & 2.50540 & -0.02580 & 1.38160 \\
$\mathrm{C}$ & 1.24060 & 0.23190 & 7.64600 \\
$\mathrm{C}$ & 2.53110 & -0.07820 & -0.00840 \\
$\mathrm{C}$ & 1.26380 & 0.10460 & 6.26180 \\
$\mathrm{C}$ & -0.00450 & 0.40600 & 9.77100 \\
$\mathrm{C}$ & 1.24640 & 0.03910 & 3.55090 \\
$\mathrm{H}$ & -0.14860 & -1.81040 & 11.84350 \\
$\mathrm{C}$ & 1.28320 & 0.01230 & 2.06850 \\
$\mathrm{C}$ & -0.73280 & 0.91790 & 11.97970 \\
$\mathrm{C}$ & 0.01360 & 0.27300 & 8.32970 \\
$\mathrm{H}$ & -1.55920 & 0.43420 & 13.91590 \\
$\mathrm{C}$ & 1.33400 & -0.09710 & -0.72660 \\
$\mathrm{C}$ & 0.05340 & 0.05580 & 5.50840 \\
$\mathrm{~N}$ & -1.09290 & 0.71210 & 10.53430 \\
$\mathrm{H}$ & -2.76470 & 0.82200 & 12.66760 \\
\hline $\mathrm{H}$ & 1.35640 & -0.16760 & -1.81020 \\
$\mathrm{~N}$ & 0.04320 & 0.00320 & 4.15220 \\
$\mathrm{C}$ & -1.82080 & 0.30850 & 12.86040 \\
$\mathrm{H}$ & -1.96730 & -0.75400 & 12.65880 \\
$\mathrm{C}$ & 0.08520 & 0.01800 & 1.33950 \\
$\mathrm{C}$ & -1.19610 & 0.19680 & 7.59320 \\
$\mathrm{C}$ & 0.11310 & -0.03640 & -0.05230 \\
$\mathrm{C}$ & -1.16580 & 0.09000 & 6.21980 \\
$\mathrm{O}$ & -2.26680 & 1.01130 & 10.13030 \\
$\mathrm{H}$ & -0.85430 & 0.06410 & 1.87640 \\
$\mathrm{H}$ & -2.13760 & 0.23900 & 8.12120 \\
$\mathrm{H}$ & -0.81990 & -0.03460 & -0.60910 \\
$\mathrm{H}$ & -2.08270 & 0.03430 & 5.64260 \\
$\mathrm{H}$ & 0.07520 & 1.96190 & 12.56350 \\
2.90900 & 11.57010 \\
& -0.6800 & 2.67480 & 13.24280 \\
& 2.43730 & 12.19620 \\
& &
\end{tabular}


Table S5: Cartesian coordinates of [6]CPPM-

\begin{tabular}{|c|c|c|c|}
\hline C & 13.429720 & 7.568790 & -3.687620 \\
\hline $\mathrm{C}$ & 13.256220 & 19.078060 & -3.928700 \\
\hline $\mathrm{C}$ & 12.183570 & 8.242740 & -3.528640 \\
\hline $\mathrm{C}$ & 12.030840 & 18.374110 & -3.740860 \\
\hline C & 14.655100 & 8.272740 & -3.875460 \\
\hline C & 14.502370 & 18.404100 & -4.087670 \\
\hline C & 10.953200 & 7.628620 & -3.826460 \\
\hline $\mathrm{C}$ & 10.782710 & 18.937880 & -4.063350 \\
\hline C & 15.903230 & 7.708970 & -3.552960 \\
\hline C & 15.732740 & 19.018230 & -3.789850 \\
\hline $\mathrm{C}$ & 9.764600 & 8.311330 & -3.770130 \\
\hline $\mathrm{C}$ & 9.615180 & 18.222630 & -3.977740 \\
\hline $\mathrm{C}$ & 17.070760 & 8.424220 & -3.638580 \\
\hline $\mathrm{C}$ & 16.921340 & 18.335520 & -3.846180 \\
\hline $\mathrm{C}$ & 9.682910 & 9.655600 & -3.356260 \\
\hline C & 9.573770 & 16.895000 & -3.507900 \\
\hline C & 17.112170 & 9.751850 & -4.108420 \\
\hline $\mathrm{C}$ & 17.003030 & 16.991250 & -4.260060 \\
\hline $\mathrm{C}$ & 10.899710 & 10.213990 & -2.923540 \\
\hline $\mathrm{C}$ & 10.806580 & 16.392130 & -3.052950 \\
\hline $\mathrm{C}$ & 15.879360 & 10.254720 & -4.563370 \\
\hline $\mathrm{C}$ & 15.786230 & 16.432850 & -4.692780 \\
\hline C & 12.081260 & 9.548810 & -3.008140 \\
\hline C & 11.967590 & 17.088490 & -3.166070 \\
\hline $\mathrm{C}$ & 14.718350 & 9.558360 & -4.450240 \\
\hline $\mathrm{C}$ & 14.604680 & 17.098040 & -4.608170 \\
\hline $\mathrm{C}$ & 8.461260 & 10.388140 & -3.367770 \\
\hline $\mathrm{C}$ & 8.374760 & 16.126150 & -3.487960 \\
\hline C & 18.311180 & 10.520700 & -4.128360 \\
\hline $\mathrm{C}$ & 18.224680 & 16.258710 & -4.248550 \\
\hline C & 8.429850 & 11.812670 & -3.431970 \\
\hline C & 8.386330 & 14.699880 & -3.492450 \\
\hline $\mathrm{C}$ & 18.299610 & 11.946970 & -4.123870 \\
\hline $\mathrm{C}$ & 18.256090 & 14.834180 & -4.184350 \\
\hline $\mathrm{C}$ & 9.443980 & 12.578100 & -4.036970 \\
\hline $\mathrm{C}$ & 9.423440 & 13.940700 & -4.065520 \\
\hline $\mathrm{C}$ & 17.262500 & 12.706150 & -3.550800 \\
\hline C & 17.241960 & 14.068750 & -3.579340 \\
\hline C & 7.336960 & 12.569490 & -2.981920 \\
\hline $\mathrm{C}$ & 7.316450 & 13.929980 & -3.010420 \\
\hline $\mathrm{C}$ & 19.369490 & 12.716870 & -4.605900 \\
\hline $\mathrm{C}$ & 19.348980 & 14.077360 & -4.634390 \\
\hline $\mathrm{C}$ & 13.452140 & 6.081650 & -3.656470 \\
\hline C & 13.233800 & 20.565200 & -3.959850 \\
\hline $\mathrm{C}$ & 13.460320 & 5.357380 & -4.860100 \\
\hline $\mathrm{C}$ & 13.220910 & 21.238360 & -5.192750 \\
\hline C & 13.465030 & 5.408490 & -2.423560 \\
\hline $\mathrm{C}$ & 13.225630 & 21.289470 & -2.756210 \\
\hline C & 13.427280 & 6.069390 & -6.177750 \\
\hline $\mathrm{C}$ & 13.210170 & 20.471140 & -6.479420 \\
\hline $\mathrm{C}$ & 13.475770 & 6.175710 & -1.136890 \\
\hline $\mathrm{C}$ & 13.258660 & 20.577460 & -1.438560 \\
\hline $\mathrm{C}$ & 13.487200 & 3.967590 & -4.797770 \\
\hline $\mathrm{C}$ & 13.205860 & 22.629710 & -5.188670 \\
\hline C & 13.480080 & 4.017130 & -2.427640 \\
\hline C & 13.198740 & 22.679250 & -2.818550 \\
\hline C & 13.494260 & 3.287580 & -3.597940 \\
\hline $\mathrm{C}$ & 13.191680 & 23.359270 & -4.018370 \\
\hline $\mathrm{C}$ & 13.517270 & 1.761650 & -3.565980 \\
\hline C & 13.168670 & 24.885200 & -4.050340 \\
\hline C & 7.159160 & 9.655990 & -3.336500 \\
\hline C & 7.051170 & 16.819400 & -3.486550 \\
\hline C & 19.634770 & 9.827450 & -4.129770 \\
\hline $\mathrm{C}$ & 19.526780 & 16.990860 & -4.279820 \\
\hline $\mathrm{C}$ & 6.745570 & 8.961560 & -2.194580 \\
\hline C & 6.616120 & 17.548250 & -2.374450 \\
\hline C & 20.069820 & 9.098590 & -5.241870 \\
\hline C & 19.940370 & 17.685290 & -5.421730 \\
\hline $\mathrm{C}$ & 7.612680 & 8.877560 & -0.979830 \\
\hline $\mathrm{C}$ & 7.479550 & 17.709120 & -1.164820 \\
\hline C & 19.206390 & 8.937730 & -6.451490 \\
\hline C & 19.073260 & 17.769290 & -6.636480 \\
\hline $\mathrm{C}$ & 5.487610 & 8.366860 & -2.187910 \\
\hline $\mathrm{C}$ & 5.340810 & 18.104530 & -2.391880 \\
\hline $\mathrm{C}$ & 21.345130 & 8.542310 & -5.224440 \\
\hline $\mathrm{C}$ & 21.198330 & 18.279990 & -5.428410 \\
\hline C & 4.627760 & 8.448430 & -3.246220 \\
\hline $\mathrm{C}$ & 4.484480 & 17.952850 & -3.445300 \\
\hline $\mathrm{C}$ & 22.201460 & 8.693990 & -4.171010 \\
\hline $\mathrm{C}$ & 22.058180 & 18.198410 & -4.370100 \\
\hline $\mathrm{C}$ & 3.231830 & 7.857680 & -3.178960 \\
\hline C & 3.071340 & 18.503580 & -3.401960 \\
\hline C & 23.614600 & 8.143270 & -4.214360 \\
\hline $\mathrm{C}$ & 23.454110 & 18.789170 & -4.437350 \\
\hline
\end{tabular}




\begin{tabular}{|c|c|c|c|}
\hline $\mathrm{C}$ & 5.068560 & 9.089400 & -4.388660 \\
\hline C & 4.945110 & 17.278190 & -4.560180 \\
\hline $\mathrm{C}$ & 21.740830 & 9.368660 & -3.056130 \\
\hline C & 21.617380 & 17.557440 & -3.227660 \\
\hline $\mathrm{C}$ & 6.322500 & 9.692950 & -4.461710 \\
\hline $\mathrm{C}$ & 6.216710 & 16.710170 & -4.608700 \\
\hline $\mathrm{C}$ & 20.469230 & 9.936670 & -3.007620 \\
\hline $\mathrm{C}$ & 20.363450 & 16.953900 & -3.154600 \\
\hline C & 6.741750 & 10.375660 & -5.738790 \\
\hline $\mathrm{C}$ & 6.657150 & 15.987540 & -5.856340 \\
\hline $\mathrm{C}$ & 20.028790 & 10.659310 & -1.759980 \\
\hline $\mathrm{C}$ & 19.944190 & 16.271190 & -1.877530 \\
\hline $\mathrm{H}$ & 10.945430 & 6.712010 & -4.076200 \\
\hline $\mathrm{H}$ & 10.747480 & 19.842570 & -4.351240 \\
\hline $\mathrm{H}$ & 15.938460 & 6.804280 & -3.265080 \\
\hline $\mathrm{H}$ & 15.740510 & 19.934830 & -3.540120 \\
\hline $\mathrm{H}$ & 8.965970 & 7.860240 & -4.018210 \\
\hline $\mathrm{H}$ & 8.803480 & 18.638660 & -4.243980 \\
\hline $\mathrm{H}$ & 17.882460 & 8.008190 & -3.372330 \\
\hline $\mathrm{H}$ & 17.719970 & 18.786610 & -3.598110 \\
\hline $\mathrm{H}$ & 10.894650 & 11.090620 & -2.557620 \\
\hline $\mathrm{H}$ & 10.827690 & 15.531840 & -2.650650 \\
\hline $\mathrm{H}$ & 15.858250 & 11.115010 & -4.965670 \\
\hline $\mathrm{H}$ & 15.791290 & 15.556230 & -5.058700 \\
\hline $\mathrm{H}$ & 13.916500 & 9.954030 & -4.771410 \\
\hline $\mathrm{H}$ & 13.815320 & 16.665280 & -4.911990 \\
\hline $\mathrm{H}$ & 12.870620 & 9.981560 & -2.704320 \\
\hline $\mathrm{H}$ & 12.769440 & 16.692810 & -2.844900 \\
\hline $\mathrm{H}$ & 10.174360 & 12.125780 & -4.442650 \\
\hline $\mathrm{H}$ & 10.140120 & 14.397440 & -4.490240 \\
\hline $\mathrm{H}$ & 16.545830 & 12.249400 & -3.126080 \\
\hline $\mathrm{H}$ & 16.511580 & 14.521070 & -3.173660 \\
\hline $\mathrm{H}$ & 6.577740 & 12.111340 & -2.641480 \\
\hline $\mathrm{H}$ & 6.543550 & 14.378890 & -2.688980 \\
\hline $\mathrm{H}$ & 20.142390 & 12.267950 & -4.927340 \\
\hline $\mathrm{H}$ & 20.108200 & 14.535510 & -4.974840 \\
\hline $\mathrm{H}$ & 13.501960 & 3.471260 & -5.607390 \\
\hline $\mathrm{H}$ & 13.206170 & 23.091940 & -6.018370 \\
\hline $\mathrm{H}$ & 13.479770 & 3.554900 & -1.597940 \\
\hline $\mathrm{H}$ & 13.183980 & 23.175590 & -2.008930 \\
\hline $\mathrm{H}$ & 5.216100 & 7.884340 & -1.415750 \\
\hline $\mathrm{H}$ & 5.054400 & 18.610560 & -1.640420 \\
\hline $\mathrm{H}$ & 21.631540 & 8.036290 & -5.975890 \\
\hline $\mathrm{H}$ & 21.469840 & 18.762510 & -6.200570 \\
\hline $\mathrm{H}$ & 4.498750 & 9.119430 & -5.148010 \\
\hline $\mathrm{H}$ & 4.376940 & 17.199240 & -5.317260 \\
\hline $\mathrm{H}$ & 22.309000 & 9.447600 & -2.299060 \\
\hline $\mathrm{H}$ & 22.187190 & 17.527410 & -2.468300 \\
\hline $\mathrm{H}$ & 13.512480 & 5.416880 & -6.903820 \\
\hline $\mathrm{H}$ & 13.276130 & 21.094950 & -7.232220 \\
\hline $\mathrm{H}$ & 13.409810 & 5.551900 & -0.384100 \\
\hline $\mathrm{H}$ & 13.173460 & 21.229970 & -0.712500 \\
\hline $\mathrm{H}$ & 14.170140 & 6.707160 & -6.222790 \\
\hline $\mathrm{H}$ & 13.971940 & 19.854720 & -6.498180 \\
\hline $\mathrm{H}$ & 12.714000 & 6.792130 & -1.118130 \\
\hline $\mathrm{H}$ & 12.515800 & 19.939690 & -1.393530 \\
\hline $\mathrm{H}$ & 12.578180 & 6.550110 & -6.268230 \\
\hline $\mathrm{H}$ & 12.375990 & 19.961690 & -6.549160 \\
\hline $\mathrm{H}$ & 14.309950 & 6.685160 & -1.067160 \\
\hline $\mathrm{H}$ & 14.107760 & 20.096740 & -1.348090 \\
\hline $\mathrm{H}$ & 14.081240 & 1.428240 & -4.294740 \\
\hline $\mathrm{H}$ & 13.722810 & 25.204650 & -4.792770 \\
\hline $\mathrm{H}$ & 12.605700 & 1.418970 & -3.675250 \\
\hline $\mathrm{H}$ & 12.247270 & 25.195380 & -4.173290 \\
\hline $\mathrm{H}$ & 13.879620 & 1.458450 & -2.707430 \\
\hline $\mathrm{H}$ & 13.521180 & 25.234850 & -3.205460 \\
\hline $\mathrm{H}$ & 7.109710 & 8.463050 & -0.247960 \\
\hline $\mathrm{H}$ & 6.963850 & 18.138560 & -0.450630 \\
\hline $\mathrm{H}$ & 19.722090 & 8.508290 & -7.165680 \\
\hline $\mathrm{H}$ & 19.576230 & 18.183800 & -7.368350 \\
\hline $\mathrm{H}$ & 7.892120 & 9.778580 & -0.715150 \\
\hline $\mathrm{H}$ & 7.785840 & 16.828790 & -0.862830 \\
\hline $\mathrm{H}$ & 18.900100 & 9.818050 & -6.753490 \\
\hline $\mathrm{H}$ & 18.793820 & 16.868260 & -6.901160 \\
\hline $\mathrm{H}$ & 8.404080 & 8.335470 & -1.180420 \\
\hline $\mathrm{H}$ & 8.254380 & 18.265930 & -1.388430 \\
\hline $\mathrm{H}$ & 18.431560 & 8.380920 & -6.227880 \\
\hline $\mathrm{H}$ & 18.281860 & 18.311370 & -6.435890 \\
\hline $\mathrm{H}$ & 3.291610 & 6.899070 & -2.985440 \\
\hline $\mathrm{H}$ & 3.102080 & 19.470810 & -3.248780 \\
\hline $\mathrm{H}$ & 23.583850 & 7.176030 & -4.367540 \\
\hline $\mathrm{H}$ & 23.394330 & 19.747780 & -4.630870 \\
\hline $\mathrm{H}$ & 2.780040 & 7.989420 & -4.039250 \\
\hline
\end{tabular}




\begin{tabular}{|c|c|c|c|}
\hline $\mathrm{H}$ & 2.624270 & 18.322380 & -4.255690 \\
\hline $\mathrm{H}$ & 24.061670 & 8.324470 & -3.360620 \\
\hline $\mathrm{H}$ & 23.905900 & 18.657420 & -3.577060 \\
\hline $\mathrm{H}$ & 2.721970 & 8.303370 & -2.471080 \\
\hline $\mathrm{H}$ & 2.574690 & 18.072760 & -2.675720 \\
\hline $\mathrm{H}$ & 24.111250 & 8.574090 & -4.940600 \\
\hline $\mathrm{H}$ & 23.963970 & 18.343480 & -5.145230 \\
\hline $\mathrm{H}$ & 6.120440 & 10.135630 & -6.457240 \\
\hline $\mathrm{H}$ & 6.029340 & 16.178450 & -6.583810 \\
\hline $\mathrm{H}$ & 20.656600 & 10.468390 & -1.032500 \\
\hline $\mathrm{H}$ & 20.565500 & 16.511220 & -1.159080 \\
\hline $\mathrm{H}$ & 7.647360 & 10.088520 & -5.980400 \\
\hline $\mathrm{H}$ & 7.553850 & 16.291460 & -6.110330 \\
\hline $\mathrm{H}$ & 19.132090 & 10.355380 & -1.505980 \\
\hline $\mathrm{H}$ & 19.038580 & 16.558320 & -1.635910 \\
\hline $\mathrm{H}$ & 6.731370 & 11.347000 & -5.608450 \\
\hline $\mathrm{H}$ & 6.675950 & 15.022640 & -5.685440 \\
\hline $\mathrm{H}$ & 20.009990 & 11.624210 & -1.930870 \\
\hline $\mathrm{H}$ & 19.954580 & 15.299850 & -2.007860 \\
\hline
\end{tabular}




\begin{tabular}{|c|c|c|c|}
\hline $\mathrm{C}$ & 2.35370 & 2.54251 & -2.19211 \\
\hline $\mathrm{C}$ & 3.07174 & 1.36823 & -2.30680 \\
\hline $\mathrm{C}$ & 4.24141 & 1.13411 & -1.55923 \\
\hline $\mathrm{C}$ & 4.73841 & 2.21904 & -0.81235 \\
\hline $\mathrm{C}$ & 4.04109 & 3.42329 & -0.73384 \\
\hline $\mathrm{C}$ & 2.77771 & 3.60520 & -1.35674 \\
\hline $\mathrm{C}$ & 1.87468 & 4.70110 & -0.96618 \\
\hline $\mathrm{C}$ & 0.24843 & 4.45367 & -2.87310 \\
\hline $\mathrm{C}$ & -1.02599 & 4.08788 & -3.16987 \\
\hline $\mathrm{C}$ & -2.06708 & 4.12904 & -2.16880 \\
\hline $\mathrm{C}$ & -1.78848 & 5.00571 & -1.05707 \\
\hline $\mathrm{C}$ & -0.50935 & 5.39461 & -0.76609 \\
\hline $\mathrm{C}$ & 0.61307 & 4.89579 & -1.54195 \\
\hline $\mathrm{C}$ & 2.23485 & 5.48772 & 0.26124 \\
\hline $\mathrm{C}$ & 2.70801 & 6.81616 & 0.11584 \\
\hline $\mathrm{C}$ & 3.06903 & 7.58233 & 1.28802 \\
\hline $\mathrm{C}$ & 2.94716 & 7.00063 & 2.55258 \\
\hline C & 2.48001 & 5.69301 & 2.71683 \\
\hline C & 2.11309 & 4.91816 & 1.55336 \\
\hline C & 2.35036 & 5.10723 & 4.01494 \\
\hline $\mathrm{C}$ & 1.87937 & 3.83151 & 4.17032 \\
\hline $\mathrm{C}$ & 1.50417 & 3.06829 & 3.02798 \\
\hline $\mathrm{C}$ & 1.61408 & 3.59289 & 1.76646 \\
\hline $\mathrm{C}$ & 2.87082 & 7.44192 & -1.16259 \\
\hline $\mathrm{C}$ & 3.33441 & 8.72647 & -1.27406 \\
\hline C & 3.67448 & 9.48129 & -0.11494 \\
\hline $\mathrm{C}$ & 3.54686 & 8.92096 & 1.12698 \\
\hline $\mathrm{H}$ & 1.38388 & 2.57401 & -2.66542 \\
\hline $\mathrm{H}$ & 2.63970 & 0.55370 & -2.87990 \\
\hline $\mathrm{H}$ & 5.66345 & 2.10864 & -0.25145 \\
\hline $\mathrm{H}$ & 4.44718 & 4.22025 & -0.11926 \\
\hline $\mathrm{H}$ & 1.01677 & 4.40526 & -3.63918 \\
\hline $\mathrm{H}$ & -1.26241 & 3.74029 & -4.17155 \\
\hline $\mathrm{H}$ & -2.58144 & 5.24039 & -0.35284 \\
\hline $\mathrm{H}$ & -0.31204 & 5.94103 & 0.14994 \\
\hline $\mathrm{H}$ & 2.63364 & 5.70368 & 4.87910 \\
\hline $\mathrm{H}$ & 1.78541 & 3.39738 & 5.16210 \\
\hline $\mathrm{H}$ & 1.12589 & 2.05814 & 3.16092 \\
\hline $\mathrm{H}$ & 1.32042 & 2.99965 & 0.90757 \\
\hline $\mathrm{H}$ & 2.61918 & 6.87741 & -2.05326 \\
\hline $\mathrm{H}$ & 3.44753 & 9.17676 & -2.25666 \\
\hline $\mathrm{H}$ & 4.03791 & 10.49960 & -0.22361 \\
\hline $\mathrm{H}$ & 3.80786 & 9.48456 & 2.01975 \\
\hline $\mathrm{C}$ & 1.34888 & -3.37316 & -1.96997 \\
\hline $\mathrm{C}$ & -0.01083 & -3.28917 & -2.18808 \\
\hline $\mathrm{C}$ & -0.92654 & -4.11275 & -1.50360 \\
\hline $\mathrm{C}$ & -0.38127 & -5.15190 & -0.72277 \\
\hline C & 0.99164 & -5.28263 & -0.54802 \\
\hline $\mathrm{C}$ & 1.91303 & -4.32816 & -1.07623 \\
\hline $\mathrm{C}$ & 3.27982 & -4.23832 & -0.60984 \\
\hline C & 4.22972 & -2.53784 & -2.25228 \\
\hline $\mathrm{C}$ & 4.49505 & -1.19296 & -2.48127 \\
\hline $\mathrm{C}$ & 4.62887 & -0.28938 & -1.40778 \\
\hline C & 4.77237 & -0.84557 & -0.12326 \\
\hline C & 4.51990 & -2.19626 & 0.10519 \\
\hline $\mathrm{C}$ & 4.08074 & -3.03817 & -0.94041 \\
\hline $\mathrm{C}$ & 3.79966 & -5.15926 & 0.44488 \\
\hline $\mathrm{C}$ & 4.81066 & -6.09380 & 0.09933 \\
\hline $\mathrm{C}$ & 5.35470 & -6.97484 & 1.10612 \\
\hline C & 4.87476 & -6.89886 & 2.41665 \\
\hline C & 3.88475 & -5.98276 & 2.78217 \\
\hline C & 3.33787 & -5.08885 & 1.78562 \\
\hline C & 3.41154 & -5.90167 & 4.12974 \\
\hline C & 2.46299 & -4.98480 & 4.49338 \\
\hline $\mathrm{C}$ & 1.93598 & -4.08697 & 3.52181 \\
\hline $\mathrm{C}$ & 2.35747 & -4.13658 & 2.21851 \\
\hline $\mathrm{C}$ & 5.31058 & -6.22166 & -1.23576 \\
\hline $\mathrm{C}$ & 6.28689 & -7.13113 & -1.54926 \\
\hline $\mathrm{C}$ & 6.82916 & -7.98778 & -0.54960 \\
\hline $\mathrm{C}$ & 6.37092 & -7.91122 & 0.73799 \\
\hline $\mathrm{H}$ & 1.97733 & -2.62431 & -2.42898 \\
\hline $\mathrm{H}$ & -0.39109 & -2.48140 & -2.80652 \\
\hline $\mathrm{H}$ & -1.04588 & -5.85198 & -0.22190 \\
\hline $\mathrm{H}$ & 1.36485 & -6.08707 & 0.07694 \\
\hline $\mathrm{H}$ & 4.04072 & -3.19382 & -3.09800 \\
\hline $\mathrm{H}$ & 4.50128 & -0.81677 & -3.50131 \\
\hline $\mathrm{H}$ & 4.92802 & -0.18859 & 0.72860 \\
\hline $\mathrm{H}$ & 4.51093 & -2.56477 & 1.12610 \\
\hline $\mathrm{H}$ & 3.83171 & -6.58653 & 4.86278 \\
\hline $\mathrm{H}$ & 2.11409 & -4.93245 & 5.52115 \\
\hline $\mathrm{H}$ & 1.19204 & -3.35287 & 3.81997 \\
\hline $\mathrm{H}$ & 1.94525 & -3.44400 & 1.49324 \\
\hline
\end{tabular}


Table S6 (cont.): Cartesian coordinates of

\begin{tabular}{rrrr} 
[3]CPBM-Ant & & \\
\hline $\mathrm{H}$ & 4.89679 & -5.58357 & -2.00906 \\
$\mathrm{H}$ & 6.64931 & -7.20519 & -2.57110 \\
$\mathrm{H}$ & 7.60200 & -8.70386 & -0.81568 \\
$\mathrm{H}$ & 6.77233 & -8.56485 & 1.50904 \\
$\mathrm{C}$ & -3.61600 & 0.82512 & -2.59305 \\
$\mathrm{C}$ & -2.94975 & 1.96606 & -2.91134 \\
$\mathrm{C}$ & -3.11952 & 3.18692 & -2.16884 \\
$\mathrm{C}$ & -4.26170 & 3.20485 & -1.29000 \\
$\mathrm{C}$ & -5.00416 & 2.08475 & -1.04288 \\
$\mathrm{C}$ & -4.62224 & 0.77561 & -1.54989 \\
$\mathrm{C}$ & -5.08246 & -0.40703 & -0.96294 \\
$\mathrm{C}$ & -3.99711 & -2.17628 & -2.47332 \\
$\mathrm{C}$ & -2.96481 & -3.09967 & -2.59505 \\
$\mathrm{C}$ & -2.32604 & -3.63098 & -1.45728 \\
$\mathrm{C}$ & -2.93799 & -3.38607 & -0.21465 \\
$\mathrm{C}$ & -3.96265 & -2.45279 & -0.09246 \\
$\mathrm{C}$ & -4.41387 & -1.70968 & -1.20676 \\
$\mathrm{C}$ & -6.10031 & -0.37137 & 0.13983 \\
$\mathrm{C}$ & -7.41510 & -0.82659 & -0.13369 \\
$\mathrm{C}$ & -8.40802 & -0.83578 & 0.91530 \\
$\mathrm{C}$ & -8.05816 & -0.39476 & 2.19478 \\
$\mathrm{C}$ & -6.76742 & 0.05335 & 2.48759 \\
$\mathrm{C}$ & -5.76329 & 0.06479 & 1.44649 \\
$\mathrm{C}$ & -6.41532 & 0.49509 & 3.80197 \\
$\mathrm{C}$ & -5.14616 & 0.91744 & 4.09056 \\
$\mathrm{C}$ & -4.14925 & 0.91775 & 3.07324 \\
$\mathrm{C}$ & -4.44612 & 0.50652 & 1.80019 \\
$\mathrm{C}$ & -7.81772 & -1.26753 & -1.43467 \\
$\mathrm{C}$ & -9.09578 & -1.69822 & -1.67829 \\
$\mathrm{C}$ & -10.06817 & -1.71366 & -0.63817 \\
$\mathrm{C}$ & -9.73077 & -1.29154 & 0.61951 \\
$\mathrm{H}$ & -3.32062 & -0.08217 & -3.09630 \\
$\mathrm{H}$ & -2.15791 & 1.90201 & -3.64965 \\
$\mathrm{H}$ & -4.54289 & 4.13640 & -0.80605 \\
$\mathrm{H}$ & -5.85474 & 2.15266 & -0.37346 \\
$\mathrm{H}$ & -4.44713 & -1.76727 & -3.37309 \\
$\mathrm{H}$ & -2.60055 & -3.36444 & -3.58473 \\
$\mathrm{H}$ & -2.51066 & -3.82119 & 0.68507 \\
$\mathrm{H}$ & -4.32167 & -2.19839 & 0.89888 \\
$\mathrm{H}$ & -7.18463 & 0.48330 & 4.57072 \\
$\mathrm{H}$ & -4.89187 & 1.24908 & 5.09367 \\
$\mathrm{H}$ & -3.14174 & 1.24783 & 3.31209 \\
$\mathrm{H}$ & -3.67550 & 0.51113 & 1.03744 \\
$\mathrm{H}$ & -7.08923 & -1.25069 & -2.23799 \\
$\mathrm{H}$ & -9.37467 & -2.02831 & -2.67546 \\
$\mathrm{H}$ & -11.07711 & -2.05712 & -0.85004 \\
$\mathrm{H}$ & -10.46589 & -1.29293 & 1.42110 \\
$\mathrm{H}$ & 3.22048 & 7.58067 & 3.43189 \\
$\mathrm{H}$ & 5.28584 & -7.56600 & 3.17184 \\
$\mathrm{H}$ & -8.80888 & -0.40181 & 2.98268 \\
\hline & & &
\end{tabular}




\begin{tabular}{|c|c|c|c|}
\hline $\mathrm{C}$ & 5.91347 & 1.82002 & 0.50953 \\
\hline $\mathrm{C}$ & 6.08769 & 3.26105 & 0.50608 \\
\hline $\mathrm{H}$ & 7.08988 & 3.65634 & 0.37330 \\
\hline $\mathrm{C}$ & 5.04353 & 4.12669 & 0.67217 \\
\hline $\mathrm{H}$ & 5.25463 & 5.19188 & 0.67012 \\
\hline $\mathrm{C}$ & 3.68522 & 3.67979 & 0.86357 \\
\hline $\mathrm{C}$ & 3.51937 & 2.24971 & 0.88365 \\
\hline $\mathrm{H}$ & 2.52453 & 1.83022 & 0.96225 \\
\hline $\mathrm{C}$ & 4.54939 & 1.38117 & 0.72090 \\
\hline $\mathrm{H}$ & 4.32858 & 0.32267 & 0.68631 \\
\hline $\mathrm{C}$ & -2.55843 & -4.53198 & -0.95055 \\
\hline $\mathrm{C}$ & -1.25060 & -3.99770 & -1.23581 \\
\hline $\mathrm{H}$ & -1.15286 & -2.98043 & -1.59449 \\
\hline $\mathrm{C}$ & -0.11585 & -4.72958 & -1.09974 \\
\hline $\mathrm{H}$ & 0.83627 & -4.27052 & -1.33905 \\
\hline $\mathrm{C}$ & -0.13170 & -6.12073 & -0.70050 \\
\hline $\mathrm{C}$ & -1.45215 & -6.71108 & -0.62421 \\
\hline $\mathrm{H}$ & -1.53257 & -7.77094 & -0.40158 \\
\hline $\mathrm{C}$ & -2.58828 & -5.95532 & -0.71597 \\
\hline $\mathrm{H}$ & -3.54402 & -6.44525 & -0.55587 \\
\hline $\mathrm{C}$ & 1.04970 & -6.80499 & -0.40864 \\
\hline $\mathrm{C}$ & 2.26517 & -6.01370 & -0.10371 \\
\hline $\mathrm{C}$ & 3.52662 & -6.25482 & -0.68324 \\
\hline $\mathrm{H}$ & 3.65882 & -7.11717 & -1.33114 \\
\hline $\mathrm{C}$ & 4.58799 & -5.36638 & -0.49759 \\
\hline $\mathrm{H}$ & 5.53261 & -5.55750 & -1.00080 \\
\hline $\mathrm{C}$ & 4.44916 & -4.22164 & 0.30859 \\
\hline $\mathrm{C}$ & 3.22171 & -4.04768 & 0.97507 \\
\hline $\mathrm{H}$ & 3.07593 & -3.17715 & 1.60698 \\
\hline $\mathrm{C}$ & 2.16169 & -4.91345 & 0.77207 \\
\hline $\mathrm{H}$ & 1.21582 & -4.72290 & 1.26987 \\
\hline $\mathrm{C}$ & 5.43233 & -3.11332 & 0.39161 \\
\hline $\mathrm{C}$ & 6.11983 & -2.64374 & -0.74188 \\
\hline $\mathrm{H}$ & 6.08328 & -3.21583 & -1.66526 \\
\hline C & 6.73319 & -1.39029 & -0.74548 \\
\hline $\mathrm{H}$ & 7.17983 & -1.01792 & -1.66342 \\
\hline $\mathrm{C}$ & 6.65372 & -0.54303 & 0.37445 \\
\hline $\mathrm{C}$ & 6.11968 & -1.08936 & 1.55718 \\
\hline $\mathrm{H}$ & 6.10490 & -0.48789 & 2.46236 \\
\hline $\mathrm{C}$ & 5.53325 & -2.34734 & 1.56811 \\
\hline $\mathrm{H}$ & 5.09452 & -2.72093 & 2.48912 \\
\hline $\mathrm{C}$ & 6.94197 & 0.91444 & 0.25849 \\
\hline $\mathrm{C}$ & 1.44952 & -8.28260 & 2.10510 \\
\hline $\mathrm{H}$ & 1.31455 & -7.20787 & 2.12337 \\
\hline $\mathrm{C}$ & 1.67787 & -8.95738 & 3.27649 \\
\hline $\mathrm{H}$ & 1.72899 & -8.40917 & 4.21344 \\
\hline $\mathrm{C}$ & 1.84194 & -10.37103 & 3.28217 \\
\hline $\mathrm{H}$ & 2.02437 & -10.88915 & 4.21975 \\
\hline $\mathrm{C}$ & 1.75719 & -11.07107 & 2.10890 \\
\hline $\mathrm{H}$ & 1.86725 & -12.15300 & 2.09877 \\
\hline $\mathrm{C}$ & 1.39740 & -11.11939 & -0.32838 \\
\hline $\mathrm{C}$ & 1.02964 & -11.22374 & -2.76033 \\
\hline $\mathrm{H}$ & 1.12772 & -12.30572 & -2.71032 \\
\hline $\mathrm{C}$ & 0.80684 & -10.59674 & -3.95584 \\
\hline $\mathrm{H}$ & 0.72151 & -11.17401 & -4.87248 \\
\hline $\mathrm{C}$ & 0.69523 & -9.17819 & -4.00084 \\
\hline $\mathrm{H}$ & 0.53165 & -8.68466 & -4.95518 \\
\hline $\mathrm{C}$ & 0.79699 & -8.42857 & -2.85768 \\
\hline $\mathrm{H}$ & 0.71591 & -7.34942 & -2.91445 \\
\hline $\mathrm{C}$ & 1.13100 & -8.29244 & -0.37522 \\
\hline $\mathrm{C}$ & 1.37501 & -8.96744 & 0.85090 \\
\hline $\mathrm{C}$ & 1.51497 & -10.40487 & 0.86725 \\
\hline $\mathrm{C}$ & 1.14532 & -10.47941 & -1.54441 \\
\hline $\mathrm{C}$ & 1.01085 & -9.03932 & -1.57929 \\
\hline $\mathrm{C}$ & 9.34067 & 0.64978 & 1.91768 \\
\hline $\mathrm{H}$ & 8.36164 & 0.43577 & 2.32983 \\
\hline $\mathrm{C}$ & 10.45933 & 0.46694 & 2.68882 \\
\hline $\mathrm{H}$ & 10.35969 & 0.09898 & 3.70648 \\
\hline $\mathrm{C}$ & 11.75290 & 0.76270 & 2.17404 \\
\hline $\mathrm{H}$ & 12.62919 & 0.61077 & 2.79840 \\
\hline $\mathrm{C}$ & 11.88561 & 1.24733 & 0.90061 \\
\hline $\mathrm{H}$ & 12.86667 & 1.49024 & 0.49890 \\
\hline C & 10.87035 & 1.97319 & -1.22542 \\
\hline $\mathrm{C}$ & 9.90117 & 2.71866 & -3.36263 \\
\hline $\mathrm{H}$ & 10.90168 & 2.96788 & -3.70855 \\
\hline $\mathrm{C}$ & 8.81448 & 2.90660 & -4.17269 \\
\hline $\mathrm{H}$ & 8.93789 & 3.31083 & -5.17375 \\
\hline $\mathrm{C}$ & 7.51440 & 2.56213 & -3.70528 \\
\hline $\mathrm{H}$ & 6.65699 & 2.70282 & -4.35809 \\
\hline $\mathrm{C}$ & 7.33272 & 2.05377 & -2.44526 \\
\hline $\mathrm{H}$ & 6.33605 & 1.79469 & -2.10750 \\
\hline $\mathrm{C}$ & 8.28481 & 1.32765 & -0.24236 \\
\hline $\mathrm{C}$ & 9.43043 & 1.13153 & 0.57367 \\
\hline
\end{tabular}


Table S7 (cont.): Cartesian coordinates of [4]CPBM-Ant

\begin{tabular}{|c|c|c|c|}
\hline $\mathrm{C}$ & 10.74297 & 1.45458 & 0.06633 \\
\hline $\mathrm{C}$ & 9.75716 & 2.18772 & -2.04229 \\
\hline $\mathrm{C}$ & 8.43677 & 1.85648 & -1.55281 \\
\hline $\mathrm{C}$ & -5.91347 & -1.82002 & -0.50953 \\
\hline $\mathrm{C}$ & -6.08769 & -3.26105 & -0.50608 \\
\hline $\mathrm{H}$ & -7.08988 & -3.65634 & -0.37330 \\
\hline $\mathrm{C}$ & -5.04353 & -4.12669 & -0.67217 \\
\hline $\mathrm{H}$ & -5.25463 & -5.19188 & -0.67012 \\
\hline $\mathrm{C}$ & -3.68522 & -3.67979 & -0.86357 \\
\hline $\mathrm{C}$ & -3.51937 & -2.24971 & -0.88365 \\
\hline $\mathrm{H}$ & -2.52453 & -1.83022 & -0.96225 \\
\hline $\mathrm{C}$ & -4.54939 & -1.38117 & -0.72090 \\
\hline $\mathrm{H}$ & -4.32858 & -0.32267 & -0.68631 \\
\hline $\mathrm{C}$ & 2.55843 & 4.53198 & 0.95055 \\
\hline $\mathrm{C}$ & 1.25060 & 3.99770 & 1.23581 \\
\hline $\mathrm{H}$ & 1.15286 & 2.98043 & 1.59449 \\
\hline $\mathrm{C}$ & 0.11585 & 4.72958 & 1.09974 \\
\hline $\mathrm{H}$ & -0.83627 & 4.27052 & 1.33905 \\
\hline $\mathrm{C}$ & 0.13170 & 6.12073 & 0.70050 \\
\hline $\mathrm{C}$ & 1.45215 & 6.71108 & 0.62421 \\
\hline $\mathrm{H}$ & 1.53257 & 7.77094 & 0.40158 \\
\hline $\mathrm{C}$ & 2.58828 & 5.95532 & 0.71597 \\
\hline $\mathrm{H}$ & 3.54402 & 6.44525 & 0.55587 \\
\hline $\mathrm{C}$ & -1.04970 & 6.80499 & 0.40864 \\
\hline $\mathrm{C}$ & -2.26517 & 6.01370 & 0.10371 \\
\hline $\mathrm{C}$ & -3.52662 & 6.25482 & 0.68324 \\
\hline $\mathrm{H}$ & -3.65882 & 7.11717 & 1.33114 \\
\hline $\mathrm{C}$ & -4.58799 & 5.36638 & 0.49759 \\
\hline $\mathrm{H}$ & -5.53261 & 5.55750 & 1.00080 \\
\hline $\mathrm{C}$ & -4.44916 & 4.22164 & -0.30859 \\
\hline $\mathrm{C}$ & -3.22171 & 4.04768 & -0.97507 \\
\hline $\mathrm{H}$ & -3.07593 & 3.17715 & -1.60698 \\
\hline $\mathrm{C}$ & -2.16169 & 4.91345 & -0.77207 \\
\hline $\mathrm{H}$ & -1.21582 & 4.72290 & -1.26987 \\
\hline $\mathrm{C}$ & -5.43233 & 3.11332 & -0.39161 \\
\hline $\mathrm{C}$ & -6.11983 & 2.64374 & 0.74188 \\
\hline $\mathrm{H}$ & -6.08328 & 3.21583 & 1.66526 \\
\hline $\mathrm{C}$ & -6.73319 & 1.39029 & 0.74548 \\
\hline $\mathrm{H}$ & -7.17983 & 1.01792 & 1.66342 \\
\hline $\mathrm{C}$ & -6.65372 & 0.54303 & -0.37445 \\
\hline $\mathrm{C}$ & -6.11968 & 1.08936 & -1.55718 \\
\hline $\mathrm{H}$ & -6.10490 & 0.48789 & -2.46236 \\
\hline $\mathrm{C}$ & -5.53325 & 2.34734 & -1.56811 \\
\hline $\mathrm{H}$ & -5.09452 & 2.72093 & -2.48912 \\
\hline $\mathrm{C}$ & -6.94197 & -0.91444 & -0.25849 \\
\hline C & -1.44952 & 8.28260 & -2.10510 \\
\hline $\mathrm{H}$ & -1.31455 & 7.20787 & -2.12337 \\
\hline $\mathrm{C}$ & -1.67787 & 8.95738 & -3.27649 \\
\hline $\mathrm{H}$ & -1.72899 & 8.40917 & -4.21344 \\
\hline $\mathrm{C}$ & -1.84194 & 10.37103 & -3.28217 \\
\hline $\mathrm{H}$ & -2.02437 & 10.88915 & -4.21975 \\
\hline $\mathrm{C}$ & -1.75719 & 11.07107 & -2.10890 \\
\hline $\mathrm{H}$ & -1.86725 & 12.15300 & -2.09877 \\
\hline $\mathrm{C}$ & -1.39740 & 11.11939 & 0.32838 \\
\hline $\mathrm{C}$ & -1.02964 & 11.22374 & 2.76033 \\
\hline $\mathrm{H}$ & -1.12772 & 12.30572 & 2.71032 \\
\hline $\mathrm{C}$ & -0.80684 & 10.59674 & 3.95584 \\
\hline $\mathrm{H}$ & -0.72151 & 11.17401 & 4.87248 \\
\hline $\mathrm{C}$ & -0.69523 & 9.17819 & 4.00084 \\
\hline $\mathrm{H}$ & -0.53165 & 8.68466 & 4.95518 \\
\hline $\mathrm{C}$ & -0.79699 & 8.42857 & 2.85768 \\
\hline $\mathrm{H}$ & -0.71591 & 7.34942 & 2.91445 \\
\hline $\mathrm{C}$ & -1.13100 & 8.29244 & 0.37522 \\
\hline $\mathrm{C}$ & -1.37501 & 8.96744 & -0.85090 \\
\hline $\mathrm{C}$ & -1.51497 & 10.40487 & -0.86725 \\
\hline $\mathrm{C}$ & -1.14532 & 10.47941 & 1.54441 \\
\hline $\mathrm{C}$ & -1.01085 & 9.03932 & 1.57929 \\
\hline $\mathrm{C}$ & -9.34067 & -0.64978 & -1.91768 \\
\hline $\mathrm{H}$ & -8.36164 & -0.43577 & -2.32983 \\
\hline $\mathrm{C}$ & -10.45933 & -0.46694 & -2.68882 \\
\hline $\mathrm{H}$ & -10.35969 & -0.09898 & -3.70648 \\
\hline $\mathrm{C}$ & -11.75290 & -0.76270 & -2.17404 \\
\hline $\mathrm{H}$ & -12.62919 & -0.61077 & -2.79840 \\
\hline $\mathrm{C}$ & -11.88561 & -1.24733 & -0.90061 \\
\hline $\mathrm{H}$ & -12.86667 & -1.49024 & -0.49890 \\
\hline $\mathrm{C}$ & -10.87035 & -1.97319 & 1.22542 \\
\hline $\mathrm{C}$ & -9.90117 & -2.71866 & 3.36263 \\
\hline $\mathrm{H}$ & -10.90168 & -2.96788 & 3.70855 \\
\hline $\mathrm{C}$ & -8.81448 & -2.90660 & 4.17269 \\
\hline $\mathrm{H}$ & -8.93789 & -3.31083 & 5.17375 \\
\hline $\mathrm{C}$ & -7.51440 & -2.56213 & 3.70528 \\
\hline $\mathrm{H}$ & -6.65699 & -2.70282 & 4.35809 \\
\hline $\mathrm{C}$ & -7.33272 & -2.05377 & 2.44526 \\
\hline
\end{tabular}


Table S7 (cont.): Cartesian coordinates of [4]CPBM-Ant

\begin{tabular}{crrr} 
4] CPBM-Ant & & \\
\hline $\mathrm{H}$ & -6.33605 & -1.79469 & 2.10750 \\
$\mathrm{C}$ & -8.28481 & -1.32765 & 0.24236 \\
$\mathrm{C}$ & -9.43043 & -1.13153 & -0.57367 \\
$\mathrm{C}$ & -10.74297 & -1.45458 & -0.06633 \\
$\mathrm{C}$ & -9.75716 & -2.18772 & 2.04229 \\
$\mathrm{C}$ & -8.43677 & -1.85648 & 1.55281 \\
$\mathrm{H}$ & 1.50257 & -12.20242 & -0.31138 \\
$\mathrm{H}$ & 11.86098 & 2.21839 & -1.60338 \\
$\mathrm{H}$ & -1.50257 & 12.20242 & 0.31138 \\
$\mathrm{H}$ & -11.86098 & -2.21839 & 1.60338
\end{tabular}




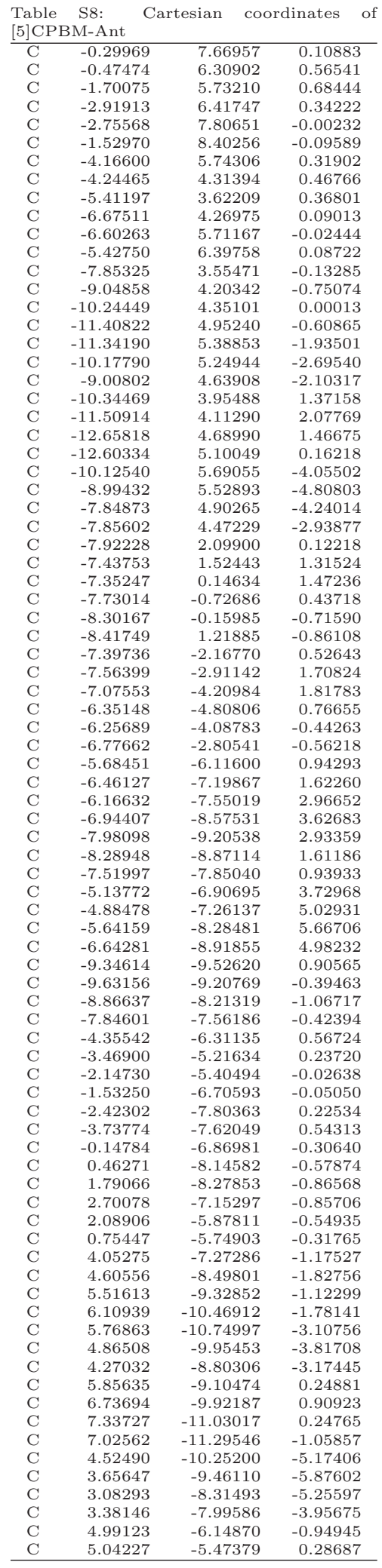




\begin{tabular}{|c|c|c|c|}
\hline $\mathrm{C}$ & 5.84552 & -4.35506 & 0.46734 \\
\hline C & 6.61459 & -3.83624 & -0.58919 \\
\hline $\mathrm{C}$ & 6.61313 & -4.54060 & -1.80558 \\
\hline C & 5.84053 & -5.68656 & -1.97395 \\
\hline $\mathrm{C}$ & 7.28869 & -2.52718 & -0.42462 \\
\hline $\mathrm{C}$ & 8.02698 & -2.20887 & 0.72962 \\
\hline C & 8.48421 & -0.91667 & 0.96038 \\
\hline C & 8.17469 & 0.13631 & 0.07071 \\
\hline C & 7.50745 & -0.20854 & -1.12767 \\
\hline C & 7.09106 & -1.50946 & -1.37522 \\
\hline $\mathrm{C}$ & 8.46225 & 1.52945 & 0.42343 \\
\hline $\mathrm{C}$ & 9.71963 & 1.84364 & 1.15354 \\
\hline C & 9.67847 & 2.24746 & 2.51748 \\
\hline C & 10.90945 & 2.52735 & 3.22150 \\
\hline $\mathrm{C}$ & 12.13055 & 2.38961 & 2.55581 \\
\hline $\mathrm{C}$ & 12.19592 & 1.98857 & 1.21836 \\
\hline $\mathrm{C}$ & 10.97431 & 1.71183 & 0.49861 \\
\hline C & 8.45737 & 2.34760 & 3.25887 \\
\hline C & 8.44985 & 2.72264 & 4.57758 \\
\hline C & 9.66397 & 3.02109 & 5.25796 \\
\hline $\mathrm{C}$ & 10.85733 & 2.92098 & 4.59566 \\
\hline $\mathrm{C}$ & 13.45003 & 1.85642 & 0.54442 \\
\hline $\mathrm{C}$ & 13.50933 & 1.48697 & -0.77221 \\
\hline C & 12.30796 & 1.23628 & -1.49351 \\
\hline C & 11.08646 & 1.34912 & -0.88147 \\
\hline C & 7.47603 & 2.55953 & 0.18549 \\
\hline $\mathrm{C}$ & 6.10041 & 2.24373 & 0.02019 \\
\hline $\mathrm{C}$ & 5.14278 & 3.22750 & -0.12891 \\
\hline C & 5.47534 & 4.59840 & -0.13524 \\
\hline $\mathrm{C}$ & 6.83944 & 4.92330 & 0.02044 \\
\hline $\mathrm{C}$ & 7.80682 & 3.94175 & 0.18074 \\
\hline $\mathrm{C}$ & 4.40267 & 5.60480 & -0.24248 \\
\hline $\mathrm{C}$ & 3.20774 & 5.29694 & -0.92413 \\
\hline $\mathrm{C}$ & 2.12069 & 6.15354 & -0.89964 \\
\hline C & 2.16135 & 7.38211 & -0.20244 \\
\hline $\mathrm{C}$ & 3.39487 & 7.74149 & 0.38210 \\
\hline C & 4.47854 & 6.86904 & 0.37561 \\
\hline $\mathrm{C}$ & 0.96350 & 8.23140 & -0.09745 \\
\hline $\mathrm{C}$ & 1.14660 & 9.71142 & -0.20635 \\
\hline C & 1.03567 & 10.52929 & 0.94876 \\
\hline C & 1.24625 & 11.95621 & 0.83992 \\
\hline C & 1.56022 & 12.51236 & -0.40293 \\
\hline $\mathrm{C}$ & 1.67577 & 11.72431 & -1.55179 \\
\hline $\mathrm{C}$ & 1.46544 & 10.29806 & -1.45838 \\
\hline C & 0.75306 & 9.99928 & 2.24951 \\
\hline C & 0.66201 & 10.81378 & 3.34808 \\
\hline C & 0.85111 & 12.22023 & 3.23083 \\
\hline C & 1.13759 & 12.77020 & 2.01092 \\
\hline C & 1.98739 & 12.30210 & -2.82207 \\
\hline $\mathrm{C}$ & 2.07869 & 11.52818 & -3.94744 \\
\hline C & 1.85465 & 10.12466 & -3.86435 \\
\hline $\mathrm{C}$ & 1.55581 & 9.53304 & -2.66450 \\
\hline $\mathrm{H}$ & 0.39976 & 5.74552 & 0.86882 \\
\hline $\mathrm{H}$ & -1.74731 & 4.72555 & 1.08322 \\
\hline $\mathrm{H}$ & -3.63219 & 8.40047 & -0.23992 \\
\hline $\mathrm{H}$ & -1.46544 & 9.44342 & -0.39678 \\
\hline $\mathrm{H}$ & -3.33519 & 3.74087 & 0.60245 \\
\hline $\mathrm{H}$ & -5.37997 & 2.54122 & 0.42867 \\
\hline $\mathrm{H}$ & -7.52291 & 6.26123 & -0.19331 \\
\hline $\mathrm{H}$ & -5.45786 & 7.47964 & 0.00878 \\
\hline $\mathrm{H}$ & -9.47705 & 3.52078 & 1.85479 \\
\hline $\mathrm{H}$ & -11.55711 & 3.79971 & 3.11726 \\
\hline $\mathrm{H}$ & -13.57235 & 4.80753 & 2.04237 \\
\hline $\mathrm{H}$ & -13.47014 & 5.55196 & -0.31493 \\
\hline $\mathrm{H}$ & -11.01279 & 6.15520 & -4.47865 \\
\hline $\mathrm{H}$ & -8.96754 & 5.86715 & -5.84039 \\
\hline $\mathrm{H}$ & -6.95941 & 4.76235 & -4.84888 \\
\hline $\mathrm{H}$ & -6.97643 & 3.99275 & -2.52550 \\
\hline $\mathrm{H}$ & -7.09618 & 2.17368 & 2.11678 \\
\hline $\mathrm{H}$ & -6.92377 & -0.26137 & 2.38387 \\
\hline $\mathrm{H}$ & -8.64653 & -0.81057 & -1.51536 \\
\hline $\mathrm{H}$ & -8.84116 & 1.62802 & -1.77358 \\
\hline $\mathrm{H}$ & -8.07633 & -2.46096 & 2.55462 \\
\hline $\mathrm{H}$ & -7.20503 & -4.75355 & 2.74890 \\
\hline $\mathrm{H}$ & -5.74888 & -4.53794 & -1.29098 \\
\hline $\mathrm{H}$ & -6.64733 & -2.26165 & -1.49413 \\
\hline $\mathrm{H}$ & -4.55214 & -6.12297 & 3.26319 \\
\hline $\mathrm{H}$ & -4.09817 & -6.75479 & 5.58225 \\
\hline $\mathrm{H}$ & -5.42429 & -8.55329 & 6.69733 \\
\hline $\mathrm{H}$ & -7.23565 & -9.69635 & 5.45837 \\
\hline $\mathrm{H}$ & -9.91825 & -10.29000 & 1.42743 \\
\hline $\mathrm{H}$ & -10.43663 & -9.71447 & -0.91993 \\
\hline $\mathrm{H}$ & -9.09288 & -7.97369 & -2.10270 \\
\hline
\end{tabular}




\begin{tabular}{|c|c|c|c|}
\hline $\mathrm{H}$ & -7.26538 & -6.81340 & -0.95125 \\
\hline $\mathrm{H}$ & -3.85782 & -4.20536 & 0.25087 \\
\hline $\mathrm{H}$ & -1.54885 & -4.52825 & -0.24528 \\
\hline $\mathrm{H}$ & -2.03304 & -8.81632 & 0.22444 \\
\hline $\mathrm{H}$ & -4.35537 & -8.48471 & 0.76567 \\
\hline $\mathrm{H}$ & -0.15644 & -9.03707 & -0.59467 \\
\hline $\mathrm{H}$ & 2.19168 & -9.26205 & -1.08889 \\
\hline $\mathrm{H}$ & 2.70732 & -4.98852 & -0.55249 \\
\hline $\mathrm{H}$ & 0.37314 & -4.75585 & -0.11048 \\
\hline $\mathrm{H}$ & 5.39905 & -8.27250 & 0.77132 \\
\hline $\mathrm{H}$ & 6.97714 & -9.72675 & 1.95098 \\
\hline $\mathrm{H}$ & 8.03432 & -11.66617 & 0.78654 \\
\hline $\mathrm{H}$ & 7.46652 & -12.14639 & -1.57283 \\
\hline $\mathrm{H}$ & 4.97872 & -11.12514 & -5.63722 \\
\hline $\mathrm{H}$ & 3.40676 & -9.69844 & -6.90659 \\
\hline $\mathrm{H}$ & 4.42598 & -5.82748 & 1.10877 \\
\hline $\mathrm{H}$ & 5.83494 & -3.83606 & 1.42204 \\
\hline $\mathrm{H}$ & 7.22373 & -4.18229 & -2.63049 \\
\hline $\mathrm{H}$ & 5.85225 & -6.20520 & -2.92829 \\
\hline $\mathrm{H}$ & 8.22965 & -2.98651 & 1.46150 \\
\hline $\mathrm{H}$ & 9.03191 & -0.69499 & 1.87148 \\
\hline $\mathrm{H}$ & 7.29621 & 0.56870 & -1.85620 \\
\hline $\mathrm{H}$ & 6.54210 & -1.73213 & -2.28650 \\
\hline $\mathrm{H}$ & 7.52290 & 2.11909 & 2.76023 \\
\hline $\mathrm{H}$ & 7.50651 & 2.79005 & 5.11297 \\
\hline $\mathrm{H}$ & 9.63741 & 3.31819 & 6.30286 \\
\hline $\mathrm{H}$ & 11.79487 & 3.13165 & 5.10501 \\
\hline $\mathrm{H}$ & 14.35901 & 2.06142 & 1.10521 \\
\hline $\mathrm{H}$ & 14.46850 & 1.39088 & -1.27380 \\
\hline $\mathrm{H}$ & 12.36286 & 0.95865 & -2.54278 \\
\hline $\mathrm{H}$ & 10.18066 & 1.16718 & -1.44814 \\
\hline $\mathrm{H}$ & 5.78173 & 1.20895 & 0.07122 \\
\hline $\mathrm{H}$ & 4.09990 & 2.93120 & -0.18391 \\
\hline $\mathrm{H}$ & 7.14824 & 5.96499 & -0.00557 \\
\hline $\mathrm{H}$ & 8.84709 & 4.23316 & 0.29012 \\
\hline $\mathrm{H}$ & 3.13647 & 4.37377 & -1.49128 \\
\hline $\mathrm{H}$ & 1.21771 & 5.88132 & -1.43736 \\
\hline $\mathrm{H}$ & 3.47781 & 8.69167 & 0.90157 \\
\hline $\mathrm{H}$ & 5.38162 & 7.14890 & 0.91106 \\
\hline $\mathrm{H}$ & 0.61243 & 8.92982 & 2.35752 \\
\hline $\mathrm{H}$ & 0.44628 & 10.38284 & 4.32213 \\
\hline $\mathrm{H}$ & 0.77291 & 12.84976 & 4.11307 \\
\hline $\mathrm{H}$ & 1.29326 & 13.84161 & 1.90731 \\
\hline $\mathrm{H}$ & 2.14664 & 13.37674 & -2.87350 \\
\hline $\mathrm{H}$ & 2.31490 & 11.97992 & -4.90709 \\
\hline $\mathrm{H}$ & 1.91782 & 9.51893 & -4.76439 \\
\hline $\mathrm{H}$ & 1.37896 & 8.46466 & -2.61833 \\
\hline $\mathrm{H}$ & -12.21915 & 5.84661 & -2.38769 \\
\hline $\mathrm{H}$ & -8.56369 & -9.97572 & 3.43518 \\
\hline $\mathrm{H}$ & 6.21624 & -11.61137 & -3.59952 \\
\hline $\mathrm{H}$ & 13.05388 & 2.59608 & 3.09354 \\
\hline $\mathrm{H}$ & 1.71821 & 13.58646 & -0.47893 \\
\hline $\mathrm{H}$ & 2.40287 & -7.68459 & -5.82279 \\
\hline $\mathrm{H}$ & 2.93888 & -7.11682 & -3.50285 \\
\hline
\end{tabular}




\begin{tabular}{|c|c|c|c|}
\hline $\mathrm{C}$ & 6.90630 & 6.24644 & -0.96826 \\
\hline $\mathrm{C}$ & 5.71842 & 5.46204 & -0.71827 \\
\hline $\mathrm{C}$ & 4.46331 & 5.97215 & -0.86253 \\
\hline $\mathrm{C}$ & 4.21307 & 7.31596 & -1.30976 \\
\hline $\mathrm{C}$ & 5.39530 & 8.09358 & -1.57622 \\
\hline $\mathrm{C}$ & 6.65665 & 7.60338 & -1.39761 \\
\hline $\mathrm{C}$ & 2.89805 & 7.83145 & -1.45339 \\
\hline $\mathrm{C}$ & 1.73169 & 7.06634 & -1.09965 \\
\hline $\mathrm{C}$ & 0.46449 & 7.55422 & -1.20992 \\
\hline $\mathrm{C}$ & 0.18643 & 8.87443 & -1.72976 \\
\hline $\mathrm{C}$ & 1.35605 & 9.63097 & -2.11770 \\
\hline $\mathrm{C}$ & 2.62306 & 9.14725 & -1.96835 \\
\hline C & -1.10790 & 9.36757 & -1.90298 \\
\hline $\mathrm{C}$ & -1.35625 & 10.57583 & -2.75176 \\
\hline $\mathrm{C}$ & -1.73260 & 11.80326 & -2.14916 \\
\hline $\mathrm{C}$ & -1.99640 & 12.95901 & -2.97500 \\
\hline $\mathrm{C}$ & -1.87823 & 12.85276 & -4.36394 \\
\hline $\mathrm{C}$ & -1.51133 & 11.65272 & -4.97887 \\
\hline $\mathrm{C}$ & -1.24488 & 10.48786 & -4.16432 \\
\hline C & -1.83391 & 11.96281 & -0.73035 \\
\hline $\mathrm{C}$ & -2.18885 & 13.16292 & -0.17129 \\
\hline $\mathrm{C}$ & -2.46168 & 14.29498 & -0.99066 \\
\hline $\mathrm{C}$ & -2.36374 & 14.19261 & -2.35215 \\
\hline C & -1.39999 & 11.55104 & -6.40127 \\
\hline $\mathrm{C}$ & -1.05747 & 10.36973 & -7.00132 \\
\hline C & -0.81044 & 9.21491 & -6.20568 \\
\hline $\mathrm{C}$ & -0.90225 & 9.27151 & -4.83929 \\
\hline $\mathrm{C}$ & -2.29002 & 8.70474 & -1.32074 \\
\hline C & -2.29701 & 8.21069 & 0.00188 \\
\hline $\mathrm{C}$ & -3.39812 & 7.53989 & 0.51944 \\
\hline $\mathrm{C}$ & -4.54258 & 7.30719 & -0.26371 \\
\hline $\mathrm{C}$ & -4.55918 & 7.83981 & -1.56555 \\
\hline $\mathrm{C}$ & -3.47203 & 8.54115 & -2.07325 \\
\hline $\mathrm{C}$ & -5.65418 & 6.47658 & 0.25051 \\
\hline C & -6.13615 & 6.60535 & 1.56571 \\
\hline $\mathrm{C}$ & -7.12659 & 5.76210 & 2.05688 \\
\hline $\mathrm{C}$ & -7.65101 & 4.71367 & 1.27227 \\
\hline $\mathrm{C}$ & -7.19222 & 4.61455 & -0.05946 \\
\hline $\mathrm{C}$ & -6.22926 & 5.48094 & -0.55938 \\
\hline C & -8.64051 & 3.76972 & 1.82306 \\
\hline $\mathrm{C}$ & -9.70873 & 4.33449 & 2.70394 \\
\hline C & -9.66710 & 4.10515 & 4.10541 \\
\hline $\mathrm{C}$ & -10.69105 & 4.67037 & 4.95570 \\
\hline $\mathrm{C}$ & -11.70609 & 5.44502 & 4.38785 \\
\hline $\mathrm{C}$ & -11.76035 & 5.68974 & 3.01263 \\
\hline $\mathrm{C}$ & -10.74741 & 5.12689 & 2.15010 \\
\hline $\mathrm{C}$ & -8.62341 & 3.35391 & 4.73712 \\
\hline C & -8.60753 & 3.15356 & 6.09293 \\
\hline $\mathrm{C}$ & -9.63195 & 3.69271 & 6.92174 \\
\hline $\mathrm{C}$ & -10.64004 & 4.43230 & 6.36519 \\
\hline C & -12.80641 & 6.47782 & 2.43882 \\
\hline $\mathrm{C}$ & -12.86704 & 6.69756 & 1.08917 \\
\hline $\mathrm{C}$ & -11.88112 & 6.13113 & 0.23248 \\
\hline C & -10.86200 & 5.37049 & 0.74438 \\
\hline C & -8.55972 & 2.39246 & 1.59545 \\
\hline $\mathrm{C}$ & -7.36491 & 1.75119 & 1.09544 \\
\hline $\mathrm{C}$ & -7.26820 & 0.39758 & 0.96739 \\
\hline $\mathrm{C}$ & -8.36015 & -0.48981 & 1.25743 \\
\hline $\mathrm{C}$ & -9.56432 & 0.14648 & 1.71527 \\
\hline $\mathrm{C}$ & -9.65052 & 1.49599 & 1.90697 \\
\hline $\mathrm{C}$ & -8.23259 & -1.89520 & 1.10236 \\
\hline $\mathrm{C}$ & -6.94557 & -2.52638 & 1.00384 \\
\hline $\mathrm{C}$ & -6.79797 & -3.86433 & 0.79253 \\
\hline C & -7.92678 & -4.74872 & 0.60850 \\
\hline $\mathrm{C}$ & -9.21749 & -4.12737 & 0.80745 \\
\hline $\mathrm{C}$ & -9.35667 & -2.78885 & 1.04386 \\
\hline $\mathrm{C}$ & -7.79688 & -6.10507 & 0.28537 \\
\hline $\mathrm{C}$ & -8.97727 & -7.02101 & 0.39570 \\
\hline $\mathrm{C}$ & -9.42580 & -7.45974 & 1.66778 \\
\hline C & -10.56029 & -8.35207 & 1.75945 \\
\hline $\mathrm{C}$ & -11.19721 & -8.77713 & 0.59037 \\
\hline $\mathrm{C}$ & -10.76634 & -8.36118 & -0.67286 \\
\hline C & -9.63659 & -7.46646 & -0.77780 \\
\hline C & -8.78213 & -7.07535 & 2.88866 \\
\hline C & -9.23513 & -7.51883 & 4.10379 \\
\hline $\mathrm{C}$ & -10.36455 & -8.38225 & 4.18914 \\
\hline $\mathrm{C}$ & -11.00295 & -8.78658 & 3.04812 \\
\hline $\mathrm{C}$ & -11.42605 & -8.79460 & -1.86528 \\
\hline $\mathrm{C}$ & -11.00885 & -8.36995 & -3.09793 \\
\hline $\mathrm{C}$ & -9.90463 & -7.47735 & -3.20610 \\
\hline $\mathrm{C}$ & -9.24604 & -7.03906 & -2.08687 \\
\hline $\mathrm{C}$ & -6.52731 & -6.69702 & -0.15656 \\
\hline C & -6.18210 & -8.02846 & 0.17322 \\
\hline
\end{tabular}


Table S9 (cont.): Cartesian coordinates of 6]CPBM-Ant

\begin{tabular}{|c|c|c|c|}
\hline $\mathrm{C}$ & -4.94297 & -8.56252 & 0.16179 \\
\hline $\mathrm{C}$ & -3.98123 & -7.79975 & -0.84999 \\
\hline $\mathrm{C}$ & -4.35596 & -6.50590 & -1.26046 \\
\hline C & -5.59461 & -5.97608 & -0.93883 \\
\hline $\mathrm{C}$ & -2.61246 & -8.28710 & -1.11205 \\
\hline $\mathrm{C}$ & -2.32819 & -9.59952 & -1.53398 \\
\hline $\mathrm{C}$ & -1.02770 & -9.99926 & -1.82037 \\
\hline C & 0.06802 & -9.11954 & -1.65942 \\
\hline C & -0.22527 & -7.80967 & -1.20991 \\
\hline C & -1.52470 & -7.40624 & -0.95577 \\
\hline $\mathrm{C}$ & 1.43303 & -9.49156 & -2.03687 \\
\hline C & 1.61746 & -10.41723 & -3.19281 \\
\hline C & 2.15431 & -11.71618 & -2.97381 \\
\hline $\mathrm{C}$ & 2.32701 & -12.61920 & -4.08995 \\
\hline C & 1.95644 & -12.20672 & -5.37233 \\
\hline $\mathrm{C}$ & 1.42272 & -10.93667 & -5.61020 \\
\hline $\mathrm{C}$ & 1.24975 & -10.01973 & -4.50690 \\
\hline C & 2.50402 & -12.20222 & -1.67261 \\
\hline $\mathrm{C}$ & 3.01153 & -13.46268 & -1.49188 \\
\hline C & 3.20330 & -14.33703 & -2.59881 \\
\hline $\mathrm{C}$ & 2.86486 & -13.92437 & -3.85882 \\
\hline $\mathrm{C}$ & 1.05918 & -10.52236 & -6.92936 \\
\hline $\mathrm{C}$ & 0.56364 & -9.26851 & -7.16522 \\
\hline $\mathrm{C}$ & 0.41260 & -8.35263 & -6.08626 \\
\hline $\mathrm{C}$ & 0.74734 & -8.71331 & -4.80644 \\
\hline C & 2.55625 & -8.95252 & -1.37749 \\
\hline $\mathrm{C}$ & 2.51026 & -8.47674 & -0.01961 \\
\hline $\mathrm{C}$ & 3.59236 & -7.89451 & 0.58322 \\
\hline $\mathrm{C}$ & 4.79999 & -7.62284 & -0.13292 \\
\hline $\mathrm{C}$ & 4.86937 & -8.14137 & -1.46472 \\
\hline $\mathrm{C}$ & 3.82955 & -8.82652 & -2.03519 \\
\hline $\mathrm{C}$ & 5.83092 & -6.81252 & 0.43769 \\
\hline $\mathrm{C}$ & 6.02391 & -6.70253 & 1.85135 \\
\hline $\mathrm{C}$ & 6.92450 & -5.82853 & 2.39865 \\
\hline $\mathrm{C}$ & 7.63625 & -4.87557 & 1.58988 \\
\hline $\mathrm{C}$ & 7.49650 & -5.04269 & 0.16710 \\
\hline C & 6.67723 & -5.99648 & -0.37607 \\
\hline C & 8.38565 & -3.83222 & 2.17175 \\
\hline $\mathrm{C}$ & 8.89514 & -3.96794 & 3.56989 \\
\hline C & 8.34612 & -3.17772 & 4.61622 \\
\hline $\mathrm{C}$ & 8.87004 & -3.29496 & 5.95742 \\
\hline C & 9.90724 & -4.19641 & 6.21412 \\
\hline C & 10.45407 & -4.99272 & 5.20453 \\
\hline $\mathrm{C}$ & 9.94667 & -4.88090 & 3.85499 \\
\hline $\mathrm{C}$ & 7.24904 & -2.28136 & 4.41166 \\
\hline $\mathrm{C}$ & 6.73308 & -1.53670 & 5.44073 \\
\hline $\mathrm{C}$ & 7.27123 & -1.63731 & 6.75452 \\
\hline C & 8.30717 & -2.49695 & 7.00213 \\
\hline $\mathrm{C}$ & 11.51478 & -5.91170 & 5.48031 \\
\hline $\mathrm{C}$ & 12.06397 & -6.67625 & 4.48722 \\
\hline $\mathrm{C}$ & 11.58434 & -6.55495 & 3.15217 \\
\hline C & 10.56773 & -5.68745 & 2.84719 \\
\hline C & 8.59708 & -2.55301 & 1.49751 \\
\hline C & 7.62908 & -2.01954 & 0.61131 \\
\hline C & 7.72050 & -0.72658 & 0.12778 \\
\hline $\mathrm{C}$ & 8.79010 & 0.12535 & 0.47518 \\
\hline $\mathrm{C}$ & 9.78953 & -0.42335 & 1.30609 \\
\hline $\mathrm{C}$ & 9.69117 & -1.71167 & 1.81214 \\
\hline C & 8.77946 & 1.54280 & 0.06363 \\
\hline $\mathrm{C}$ & 8.00200 & 1.98002 & -1.02895 \\
\hline $\mathrm{C}$ & 7.83483 & 3.32712 & -1.30787 \\
\hline $\mathrm{C}$ & 8.45713 & 4.32680 & -0.52744 \\
\hline $\mathrm{C}$ & 9.32893 & 3.88629 & 0.49074 \\
\hline C & 9.46620 & 2.53691 & 0.79043 \\
\hline C & 8.19788 & 5.75898 & -0.74546 \\
\hline C & 9.36256 & 6.69026 & -0.63937 \\
\hline $\mathrm{C}$ & 9.48731 & 7.55435 & 0.48132 \\
\hline $\mathrm{C}$ & 10.63659 & 8.42550 & 0.59069 \\
\hline $\mathrm{C}$ & 11.61664 & 8.40005 & -0.40508 \\
\hline $\mathrm{C}$ & 11.51589 & 7.55337 & -1.51281 \\
\hline C & 10.37054 & 6.68219 & -1.63873 \\
\hline $\mathrm{C}$ & 8.53148 & 7.58505 & 1.54832 \\
\hline $\mathrm{C}$ & 8.68460 & 8.42489 & 2.62076 \\
\hline $\mathrm{C}$ & 9.80756 & 9.29539 & 2.71154 \\
\hline C & 10.75528 & 9.28957 & 1.72435 \\
\hline C & 12.52094 & 7.53794 & -2.52975 \\
\hline C & 12.40864 & 6.72052 & -3.62195 \\
\hline $\mathrm{C}$ & 11.27279 & 5.87373 & -3.76111 \\
\hline $\mathrm{C}$ & 10.28886 & 5.85880 & -2.80664 \\
\hline $\mathrm{H}$ & 5.82969 & 4.45088 & -0.34519 \\
\hline $\mathrm{H}$ & 3.63019 & 5.32526 & -0.61356 \\
\hline $\mathrm{H}$ & 5.29410 & 9.11740 & -1.91938 \\
\hline $\mathrm{H}$ & 7.50842 & 8.24196 & -1.60884 \\
\hline
\end{tabular}




\begin{tabular}{|c|c|c|c|}
\hline $\mathrm{H}$ & 1.84595 & 6.05282 & -0.73354 \\
\hline $\mathrm{H}$ & -0.36712 & 6.91019 & -0.94848 \\
\hline $\mathrm{H}$ & 1.21488 & 10.63175 & -2.51271 \\
\hline $\mathrm{H}$ & 3.44702 & 9.78778 & -2.26351 \\
\hline $\mathrm{H}$ & -1.62103 & 11.11156 & -0.09388 \\
\hline $\mathrm{H}$ & -2.25987 & 13.25597 & 0.90915 \\
\hline $\mathrm{H}$ & -2.74152 & 15.23821 & -0.52932 \\
\hline $\mathrm{H}$ & -2.56130 & 15.05182 & -2.98898 \\
\hline $\mathrm{H}$ & -1.59956 & 12.43910 & -6.99657 \\
\hline $\mathrm{H}$ & -0.97758 & 10.30538 & -8.08312 \\
\hline $\mathrm{H}$ & -0.54791 & 8.27825 & -6.69023 \\
\hline $\mathrm{H}$ & -0.71294 & 8.38342 & -4.24721 \\
\hline $\mathrm{H}$ & -1.42273 & 8.36085 & 0.62861 \\
\hline $\mathrm{H}$ & -3.35837 & 7.15250 & 1.53419 \\
\hline $\mathrm{H}$ & -5.44419 & 7.71000 & -2.18301 \\
\hline $\mathrm{H}$ & -3.51755 & 8.93917 & -3.08258 \\
\hline $\mathrm{H}$ & -5.73478 & 7.38683 & 2.20556 \\
\hline $\mathrm{H}$ & -7.48111 & 5.88827 & 3.07549 \\
\hline $\mathrm{H}$ & -7.60660 & 3.84717 & -0.70685 \\
\hline $\mathrm{H}$ & -5.88379 & 5.36318 & -1.58298 \\
\hline $\mathrm{H}$ & -7.83310 & 2.94024 & 4.12155 \\
\hline $\mathrm{H}$ & -7.80260 & 2.57883 & 6.54331 \\
\hline $\mathrm{H}$ & -9.60412 & 3.52001 & 7.99419 \\
\hline $\mathrm{H}$ & -11.42387 & 4.85963 & 6.98642 \\
\hline $\mathrm{H}$ & -13.55801 & 6.89752 & 3.10352 \\
\hline $\mathrm{H}$ & -13.66779 & 7.29785 & 0.66578 \\
\hline $\mathrm{H}$ & -11.94216 & 6.30182 & -0.83902 \\
\hline $\mathrm{H}$ & -10.12363 & 4.93834 & 0.07854 \\
\hline $\mathrm{H}$ & -6.49959 & 2.36206 & 0.86600 \\
\hline $\mathrm{H}$ & -6.33554 & -0.01558 & 0.59852 \\
\hline $\mathrm{H}$ & -10.42572 & -0.46507 & 1.96436 \\
\hline $\mathrm{H}$ & -10.57597 & 1.92123 & 2.28165 \\
\hline $\mathrm{H}$ & -6.05223 & -1.93478 & 1.17453 \\
\hline $\mathrm{H}$ & -5.80008 & -4.28596 & 0.81087 \\
\hline $\mathrm{H}$ & -10.10579 & -4.74308 & 0.71443 \\
\hline $\mathrm{H}$ & -10.36050 & -2.38336 & 1.12319 \\
\hline $\mathrm{H}$ & -7.91825 & -6.42167 & 2.83832 \\
\hline $\mathrm{H}$ & -8.72747 & -7.21115 & 5.01405 \\
\hline $\mathrm{H}$ & -10.71090 & -8.72106 & 5.16181 \\
\hline $\mathrm{H}$ & -11.86166 & -9.45207 & 3.09893 \\
\hline $\mathrm{H}$ & -12.27279 & -9.47023 & -1.76810 \\
\hline $\mathrm{H}$ & -11.51897 & -8.70635 & -3.99646 \\
\hline $\mathrm{H}$ & -9.58570 & -7.13824 & -4.18811 \\
\hline $\mathrm{H}$ & -8.41261 & -6.35244 & -2.18386 \\
\hline $\mathrm{H}$ & -6.88197 & -8.62869 & 0.74633 \\
\hline $\mathrm{H}$ & -4.69285 & -9.56884 & 0.16411 \\
\hline $\mathrm{H}$ & -3.66933 & -5.92210 & -1.86710 \\
\hline $\mathrm{H}$ & -5.86055 & -4.99361 & -1.31423 \\
\hline $\mathrm{H}$ & -3.14470 & -10.30213 & -1.67939 \\
\hline $\mathrm{H}$ & -0.84510 & -11.00962 & -2.17444 \\
\hline $\mathrm{H}$ & 0.58210 & -7.09237 & -1.10571 \\
\hline $\mathrm{H}$ & -1.70902 & -6.38956 & -0.62010 \\
\hline $\mathrm{H}$ & 2.35940 & -11.55336 & -0.81708 \\
\hline $\mathrm{H}$ & 3.26750 & -13.80185 & -0.49167 \\
\hline $\mathrm{H}$ & 3.60959 & -15.33168 & -2.43636 \\
\hline $\mathrm{H}$ & 2.99439 & -14.58506 & -4.71298 \\
\hline $\mathrm{H}$ & 1.19206 & -11.22975 & -7.74465 \\
\hline $\mathrm{H}$ & 0.29207 & -8.96470 & -8.17256 \\
\hline $\mathrm{H}$ & 0.03243 & -7.35364 & -6.28278 \\
\hline $\mathrm{H}$ & 0.63299 & -7.99818 & -4.00006 \\
\hline $\mathrm{H}$ & 1.59692 & -8.61467 & 0.54997 \\
\hline $\mathrm{H}$ & 3.49879 & -7.55656 & 1.61095 \\
\hline $\mathrm{H}$ & 5.78909 & -8.02205 & -2.03031 \\
\hline $\mathrm{H}$ & 3.93531 & -9.21951 & -3.04140 \\
\hline $\mathrm{H}$ & 5.46278 & -7.35726 & 2.51159 \\
\hline $\mathrm{H}$ & 7.04850 & -5.79148 & 3.47605 \\
\hline $\mathrm{H}$ & 8.07956 & -4.40621 & -0.49056 \\
\hline $\mathrm{H}$ & 6.60741 & -6.07324 & -1.45716 \\
\hline $\mathrm{H}$ & 6.82031 & -2.19652 & 3.42025 \\
\hline $\mathrm{H}$ & 5.90021 & -0.86394 & 5.25433 \\
\hline $\mathrm{H}$ & 6.85056 & -1.03701 & 7.55671 \\
\hline $\mathrm{H}$ & 8.72065 & -2.59468 & 8.00327 \\
\hline $\mathrm{H}$ & 11.87701 & -5.98532 & 6.50311 \\
\hline $\mathrm{H}$ & 12.86970 & -7.37073 & 4.70928 \\
\hline $\mathrm{H}$ & 12.03462 & -7.15538 & 2.36616 \\
\hline $\mathrm{H}$ & 10.21996 & -5.60359 & 1.82423 \\
\hline $\mathrm{H}$ & 6.74741 & -2.60506 & 0.37299 \\
\hline $\mathrm{H}$ & 6.90026 & -0.34528 & -0.47193 \\
\hline $\mathrm{H}$ & 10.65979 & 0.17186 & 1.56693 \\
\hline $\mathrm{H}$ & 10.46965 & -2.08330 & 2.47181 \\
\hline $\mathrm{H}$ & 7.52162 & 1.25210 & -1.67514 \\
\hline $\mathrm{H}$ & 7.22068 & 3.62094 & -2.15433 \\
\hline $\mathrm{H}$ & 9.84886 & 4.62200 & 1.09697 \\
\hline
\end{tabular}


Table S9 (cont.): Cartesian coordinates of [6]CPBM-Ant

\begin{tabular}{crrr} 
[6]CPBM-Ant \\
H & 10.07375 & 2.25530 & 1.64511 \\
$\mathrm{H}$ & 7.67148 & 6.92721 & 1.49984 \\
$\mathrm{H}$ & 7.94211 & 8.42574 & 3.41441 \\
$\mathrm{H}$ & 9.91016 & 9.95506 & 3.56892 \\
$\mathrm{H}$ & 11.62446 & 9.94060 & 1.78476 \\
$\mathrm{H}$ & 13.37941 & 8.19584 & -2.41520 \\
$\mathrm{H}$ & 13.17922 & 6.71823 & -4.38824 \\
$\mathrm{H}$ & 11.18515 & 5.23654 & -4.63709 \\
$\mathrm{H}$ & 9.42629 & 5.21465 & -2.93361 \\
$\mathrm{H}$ & -2.07712 & 13.72646 & -4.98157 \\
$\mathrm{H}$ & -12.47369 & 5.86906 & 5.03244 \\
$\mathrm{H}$ & -12.05037 & -9.44861 & 0.66549 \\
$\mathrm{H}$ & 2.08819 & -12.89161 & -6.20776 \\
$\mathrm{H}$ & 10.29603 & -4.28257 & 7.22694 \\
$\mathrm{H}$ & 12.48161 & 9.05445 & -0.31496 \\
\hline & & &
\end{tabular}


Table S10: Cartesian coordinates of MC

\begin{tabular}{|c|c|c|c|}
\hline $3 \mathrm{~A} 3$ & & & \\
\hline $\mathrm{C}$ & -2.23867 & 3.55090 & -0.49160 \\
\hline C & -1.33400 & 4.58631 & -0.43697 \\
\hline $\mathrm{C}$ & 0.03801 & 4.30805 & -0.34049 \\
\hline $\mathrm{C}$ & 0.47370 & 2.94311 & -0.32033 \\
\hline $\mathrm{C}$ & -0.42340 & 1.91625 & -0.37916 \\
\hline C & -1.81167 & 2.19926 & -0.46122 \\
\hline $\mathrm{C}$ & 1.17853 & 5.15716 & -0.28761 \\
\hline $\mathrm{C}$ & 2.33402 & 4.32867 & -0.22941 \\
\hline C & 1.92247 & 2.95617 & -0.24129 \\
\hline $\mathrm{C}$ & 2.83743 & 1.94564 & -0.17506 \\
\hline C & 3.70082 & 4.63146 & -0.13293 \\
\hline $\mathrm{C}$ & 4.62344 & 3.61251 & -0.06924 \\
\hline $\mathrm{C}$ & 4.22033 & 2.25339 & -0.09206 \\
\hline $\mathrm{C}$ & 5.10375 & 1.18435 & -0.03070 \\
\hline $\mathrm{C}$ & -2.67647 & 1.11457 & -0.51147 \\
\hline $\mathrm{C}$ & -3.28108 & 0.05115 & -0.53106 \\
\hline C & 5.73479 & 0.13639 & -0.01663 \\
\hline C & -3.76666 & -1.24752 & -0.56448 \\
\hline C & 6.24639 & -1.15245 & 0.01252 \\
\hline $\mathrm{H}$ & -3.29728 & 3.75626 & -0.56142 \\
\hline $\mathrm{H}$ & -1.67809 & 5.61122 & -0.46004 \\
\hline $\mathrm{H}$ & 4.02685 & 5.66239 & -0.11611 \\
\hline $\mathrm{H}$ & 5.67811 & 3.83687 & 0.00163 \\
\hline C & -5.14489 & -1.57507 & -0.64198 \\
\hline C & -2.80864 & -2.29666 & -0.51887 \\
\hline C & -3.22534 & -3.59436 & -0.53966 \\
\hline C & -5.56494 & -2.88362 & -0.66811 \\
\hline $\mathrm{C}$ & -4.62039 & -3.92256 & -0.60631 \\
\hline $\mathrm{C}$ & -4.75962 & -5.33063 & -0.64247 \\
\hline C & -3.43917 & -5.90247 & -0.59146 \\
\hline C & -2.48617 & -4.84577 & -0.51882 \\
\hline C & -2.99836 & -7.22607 & -0.55664 \\
\hline C & -1.14533 & -5.10934 & -0.43370 \\
\hline C & -0.69640 & -6.45018 & -0.41677 \\
\hline C & -1.64301 & -7.49263 & -0.47563 \\
\hline $\mathrm{C}$ & 0.68670 & -6.66104 & -0.33886 \\
\hline $\mathrm{C}$ & 1.90209 & -6.64963 & -0.27141 \\
\hline $\mathrm{C}$ & 3.28061 & -6.41322 & -0.18636 \\
\hline $\mathrm{H}$ & -5.86482 & -0.77057 & -0.68489 \\
\hline $\mathrm{H}$ & -6.61950 & -3.11510 & -0.72891 \\
\hline $\mathrm{H}$ & -3.70954 & -8.03941 & -0.60101 \\
\hline $\mathrm{H}$ & -1.29267 & -8.51457 & -0.45435 \\
\hline $\mathrm{C}$ & 3.70322 & -5.06403 & -0.15669 \\
\hline C & 5.03937 & -4.77503 & -0.08251 \\
\hline C & 6.01410 & -5.81320 & -0.03520 \\
\hline C & 5.59707 & -7.14490 & -0.04722 \\
\hline C & 4.24697 & -7.43731 & -0.12756 \\
\hline C & 5.75164 & -3.50944 & -0.02076 \\
\hline C & 7.15053 & -3.81048 & 0.08164 \\
\hline C & 7.31982 & -5.21591 & 0.07144 \\
\hline $\mathrm{C}$ & 5.31034 & -2.22021 & -0.05410 \\
\hline C & 7.63007 & -1.45312 & 0.09839 \\
\hline C & 8.07538 & -2.75326 & 0.12805 \\
\hline $\mathrm{H}$ & 3.91597 & -8.46578 & -0.14327 \\
\hline $\mathrm{H}$ & 6.32354 & -7.94459 & -0.00235 \\
\hline $\mathrm{H}$ & 8.33447 & -0.63482 & 0.13814 \\
\hline $\mathrm{H}$ & 9.13393 & -2.96394 & 0.19480 \\
\hline C & 1.16477 & 6.61770 & -0.29066 \\
\hline $\mathrm{C}$ & 0.55997 & 7.31990 & 0.77368 \\
\hline $\mathrm{C}$ & 0.53674 & 8.75806 & 0.75640 \\
\hline C & 1.13545 & 9.43899 & -0.29725 \\
\hline C & 1.74873 & 8.76575 & -1.34745 \\
\hline C & 1.75566 & 7.32729 & -1.35783 \\
\hline $\mathrm{C}$ & 2.35264 & 9.46649 & -2.42368 \\
\hline C & 2.91718 & 8.79907 & -3.46916 \\
\hline C & 2.89249 & 7.38684 & -3.50116 \\
\hline C & 2.32540 & 6.67636 & -2.48358 \\
\hline C & -0.08187 & 9.45102 & 1.82927 \\
\hline C & -0.63188 & 8.77702 & 2.87824 \\
\hline C & -0.57692 & 7.36585 & 2.91737 \\
\hline C & 0.00477 & 6.66260 & 1.90303 \\
\hline $\mathrm{H}$ & -0.99322 & 6.83956 & 3.76598 \\
\hline $\mathrm{H}$ & -1.09783 & 9.31831 & 3.69084 \\
\hline $\mathrm{H}$ & -0.10076 & 10.53323 & 1.79960 \\
\hline $\mathrm{H}$ & 1.12393 & 10.52263 & -0.29995 \\
\hline $\mathrm{H}$ & 2.34856 & 10.54898 & -2.39934 \\
\hline $\mathrm{H}$ & 3.37176 & 9.34607 & -4.28437 \\
\hline $\mathrm{H}$ & 3.32039 & 6.86534 & -4.34694 \\
\hline $\mathrm{H}$ & 2.29925 & 5.59843 & -2.53068 \\
\hline $\mathrm{H}$ & 0.05390 & 5.58574 & 1.95546 \\
\hline C & -6.00931 & -6.07759 & -0.71231 \\
\hline C & -6.95610 & -5.97399 & 0.33095 \\
\hline C & -8.18829 & -6.71143 & 0.24841 \\
\hline
\end{tabular}


Table S10 (cont.): Cartesian coordinates of

\begin{tabular}{rrrr} 
MC-F3A3 & & & \\
\hline $\mathrm{C}$ & -8.42018 & -7.53622 & -0.84625 \\
$\mathrm{C}$ & -7.49588 & -7.66071 & -1.87726 \\
$\mathrm{C}$ & -6.27060 & -6.91043 & -1.82258 \\
$\mathrm{C}$ & -7.74470 & -8.49632 & -2.99664 \\
$\mathrm{C}$ & -5.38978 & -7.00055 & -2.93175 \\
$\mathrm{C}$ & -5.66702 & -7.81333 & -3.99251 \\
$\mathrm{C}$ & -6.85286 & -8.57975 & -4.02393 \\
$\mathrm{C}$ & -9.13407 & -6.59654 & 1.30006 \\
$\mathrm{C}$ & -8.88238 & -5.81888 & 2.39045 \\
$\mathrm{C}$ & -7.65620 & -5.12550 & 2.49406 \\
$\mathrm{C}$ & -6.72276 & -5.20645 & 1.50184 \\
$\mathrm{H}$ & -7.45254 & -4.53167 & 3.37507 \\
$\mathrm{H}$ & -9.61085 & -5.74242 & 3.18653 \\
$\mathrm{H}$ & -10.06135 & -7.14977 & 1.22049 \\
$\mathrm{H}$ & -9.34628 & -8.09656 & -0.89758 \\
$\mathrm{H}$ & -8.66734 & -9.06233 & -3.02123 \\
$\mathrm{H}$ & -7.05644 & -9.21827 & -4.87311 \\
$\mathrm{H}$ & -4.97697 & -7.86475 & -4.82406 \\
$\mathrm{H}$ & -4.48901 & -6.40617 & -2.93327 \\
$\mathrm{H}$ & -5.78401 & -4.68448 & 1.60544 \\
$\mathrm{C}$ & 8.58301 & -5.93745 & 0.16143 \\
$\mathrm{C}$ & 9.39296 & -5.80648 & 1.31160 \\
$\mathrm{C}$ & 10.64183 & -6.51621 & 1.38595 \\
$\mathrm{C}$ & 11.02302 & -7.34250 & 0.33511 \\
$\mathrm{C}$ & 10.23375 & -7.49491 & -0.79914 \\
$\mathrm{C}$ & 8.99506 & -6.77199 & -0.90087 \\
$\mathrm{C}$ & 8.25790 & -6.88957 & -2.10789 \\
$\mathrm{C}$ & 10.63476 & -8.33245 & -1.87190 \\
$\mathrm{C}$ & 9.87648 & -8.44317 & -2.99904 \\
$\mathrm{C}$ & 8.67942 & -7.70340 & -3.11925 \\
$\mathrm{C}$ & 9.00178 & -5.03842 & 2.43925 \\
$\mathrm{C}$ & 9.80541 & -4.93063 & 3.53698 \\
$\mathrm{C}$ & 11.05031 & -5.59582 & 3.58887 \\
$\mathrm{C}$ & 11.44996 & -6.37355 & 2.54364 \\
$\mathrm{H}$ & 9.48261 & -4.33715 & 4.38188 \\
$\mathrm{H}$ & 11.67467 & -5.49809 & 4.46685 \\
$\mathrm{H}$ & 12.39203 & -6.90577 & 2.58196 \\
$\mathrm{H}$ & 11.96054 & -7.88186 & 0.40175 \\
$\mathrm{H}$ & 11.56549 & -8.87771 & -1.77876 \\
$\mathrm{H}$ & 10.19546 & -9.08290 & -3.81097 \\
$\mathrm{H}$ & 8.09682 & -7.77615 & -4.02785 \\
$\mathrm{H}$ & 7.35141 & -6.31564 & -2.22445 \\
$\mathrm{H}$ & 8.04594 & -4.53799 & 2.42415 \\
$\mathrm{H}$ & -1.75964 & -2.03848 & -0.47158 \\
$\mathrm{H}$ & -0.41535 & -4.31375 & -0.37628 \\
$\mathrm{H}$ & 2.95693 & -4.28237 & -0.19103 \\
$\mathrm{H}$ & 4.25838 & -1.98265 & -0.13276 \\
$\mathrm{H}$ & -0.10373 & 0.88321 & -0.36561 \\
$\mathrm{H}$ & 2.53570 & 0.90714 & -0.18141 \\
\hline & & &
\end{tabular}




\begin{tabular}{|c|c|c|c|}
\hline $\mathrm{C}$ & 1.21051 & -5.96070 & -0.27010 \\
\hline C & 1.18945 & -4.73658 & 0.52615 \\
\hline $\mathrm{C}$ & 0.00000 & -4.16538 & 0.95788 \\
\hline $\mathrm{C}$ & -1.18945 & -4.73658 & 0.52615 \\
\hline $\mathrm{C}$ & -1.21051 & -5.96070 & -0.27010 \\
\hline $\mathrm{C}$ & 0.00000 & -6.59001 & -0.62294 \\
\hline C & -2.55273 & -6.16745 & -0.68738 \\
\hline C & 2.55273 & -6.16745 & -0.68738 \\
\hline $\mathrm{C}$ & 2.50821 & -4.19186 & 0.53144 \\
\hline $\mathrm{C}$ & -2.50821 & -4.19186 & 0.53144 \\
\hline $\mathrm{C}$ & 3.35594 & -5.06653 & -0.26082 \\
\hline $\mathrm{C}$ & 4.65393 & -4.65393 & -0.61725 \\
\hline C & 5.06653 & -3.35594 & -0.26083 \\
\hline C & 4.19186 & -2.50821 & 0.53144 \\
\hline $\mathrm{C}$ & 2.94189 & -2.94189 & 0.95297 \\
\hline $\mathrm{C}$ & -2.94189 & -2.94189 & 0.95297 \\
\hline $\mathrm{C}$ & -4.19186 & -2.50821 & 0.53144 \\
\hline $\mathrm{C}$ & -5.06653 & -3.35594 & -0.26083 \\
\hline $\mathrm{C}$ & -4.65393 & -4.65393 & -0.61725 \\
\hline $\mathrm{C}$ & -3.35594 & -5.06653 & -0.26082 \\
\hline $\mathrm{C}$ & 5.96070 & 1.21052 & -0.27012 \\
\hline $\mathrm{C}$ & 4.73658 & 1.18945 & 0.52614 \\
\hline $\mathrm{C}$ & 4.16538 & 0.00000 & 0.95788 \\
\hline C & 4.73658 & -1.18945 & 0.52614 \\
\hline $\mathrm{C}$ & 5.96070 & -1.21052 & -0.27012 \\
\hline $\mathrm{C}$ & 6.59001 & 0.00000 & -0.62295 \\
\hline $\mathrm{C}$ & 6.16744 & -2.55273 & -0.68739 \\
\hline $\mathrm{C}$ & 6.16744 & 2.55273 & -0.68739 \\
\hline C & -1.21051 & 5.96070 & -0.27010 \\
\hline C & -1.18945 & 4.73658 & 0.52615 \\
\hline $\mathrm{C}$ & 0.00000 & 4.16538 & 0.95788 \\
\hline $\mathrm{C}$ & 1.18945 & 4.73658 & 0.52615 \\
\hline $\mathrm{C}$ & 1.21051 & 5.96070 & -0.27010 \\
\hline $\mathrm{C}$ & 0.00000 & 6.59001 & -0.62294 \\
\hline $\mathrm{C}$ & 2.55273 & 6.16745 & -0.68738 \\
\hline $\mathrm{C}$ & -2.55273 & 6.16745 & -0.68738 \\
\hline $\mathrm{C}$ & -2.50821 & 4.19186 & 0.53144 \\
\hline C & 2.50821 & 4.19186 & 0.53144 \\
\hline C & -3.35594 & 5.06653 & -0.26082 \\
\hline $\mathrm{C}$ & -4.65393 & 4.65393 & -0.61725 \\
\hline $\mathrm{C}$ & -5.06653 & 3.35594 & -0.26083 \\
\hline $\mathrm{C}$ & -4.19186 & 2.50821 & 0.53144 \\
\hline $\mathrm{C}$ & -2.94189 & 2.94189 & 0.95297 \\
\hline $\mathrm{C}$ & 2.94189 & 2.94189 & 0.95297 \\
\hline $\mathrm{C}$ & 4.19186 & 2.50821 & 0.53144 \\
\hline $\mathrm{C}$ & 5.06653 & 3.35594 & -0.26083 \\
\hline $\mathrm{C}$ & 4.65393 & 4.65393 & -0.61725 \\
\hline $\mathrm{C}$ & 3.35594 & 5.06653 & -0.26082 \\
\hline C & -5.96070 & -1.21052 & -0.27012 \\
\hline $\mathrm{C}$ & -4.73658 & -1.18945 & 0.52614 \\
\hline $\mathrm{C}$ & -4.16538 & 0.00000 & 0.95788 \\
\hline $\mathrm{C}$ & -4.73658 & 1.18945 & 0.52614 \\
\hline $\mathrm{C}$ & -5.96070 & 1.21052 & -0.27012 \\
\hline $\mathrm{C}$ & -6.59001 & 0.00000 & -0.62295 \\
\hline $\mathrm{C}$ & -6.16744 & 2.55273 & -0.68739 \\
\hline $\mathrm{C}$ & -6.16744 & -2.55273 & -0.68739 \\
\hline $\mathrm{H}$ & 0.00000 & -3.23301 & 1.51594 \\
\hline $\mathrm{H}$ & 0.00000 & -7.48114 & -1.24527 \\
\hline $\mathrm{H}$ & 5.28248 & -5.28248 & -1.24311 \\
\hline $\mathrm{H}$ & 2.27957 & -2.27957 & 1.50427 \\
\hline $\mathrm{H}$ & -2.27957 & -2.27957 & 1.50427 \\
\hline $\mathrm{H}$ & -5.28248 & -5.28248 & -1.24311 \\
\hline $\mathrm{H}$ & 3.23301 & 0.00000 & 1.51593 \\
\hline $\mathrm{H}$ & 7.48113 & 0.00000 & -1.24529 \\
\hline $\mathrm{H}$ & 0.00000 & 3.23301 & 1.51594 \\
\hline $\mathrm{H}$ & 0.00000 & 7.48114 & -1.24527 \\
\hline $\mathrm{H}$ & -5.28248 & 5.28248 & -1.24311 \\
\hline $\mathrm{H}$ & -2.27957 & 2.27957 & 1.50427 \\
\hline $\mathrm{H}$ & 2.27957 & 2.27957 & 1.50427 \\
\hline $\mathrm{H}$ & 5.28248 & 5.28248 & -1.24311 \\
\hline $\mathrm{H}$ & -3.23301 & 0.00000 & 1.51593 \\
\hline $\mathrm{H}$ & -7.48113 & 0.00000 & -1.24529 \\
\hline $\mathrm{H}$ & 2.89474 & 6.98369 & -1.31343 \\
\hline $\mathrm{H}$ & -6.98368 & 2.89474 & -1.31345 \\
\hline $\mathrm{H}$ & -6.98368 & -2.89474 & -1.31345 \\
\hline $\mathrm{H}$ & -2.89474 & -6.98369 & -1.31343 \\
\hline $\mathrm{H}$ & 6.98368 & -2.89474 & -1.31345 \\
\hline $\mathrm{H}$ & 6.98368 & 2.89474 & -1.31345 \\
\hline $\mathrm{H}$ & -2.89474 & 6.98369 & -1.31343 \\
\hline $\mathrm{H}$ & 2.89474 & -6.98369 & -1.31343 \\
\hline
\end{tabular}




\section{References}

[1] Li, Z.; Gopalakrishna, T. Y.; Han, Y.; Gu, Y.; Yuan, L.; Zeng, W.; Casanova, D.; Wu, J. [6]Cyclo-para-phenylmethine: an analog of benzene showing global aromaticity and open-shell diradical character. Journal of the American Chemical Society 2019, 141, 16266-16270.

[2] Ni, Y.; Sandoval-Salinas, M. E.; Tanaka, T.; Phan, H.; Herng, T. S.; Gopalakrishna, T. Y.; Ding, J.; Osuka, A.; Casanova, D.; Wu, J. $[n]$ Cyclo-para-biphenylmethine polyradicaloids: $[n]$ annulene analogs and unusual valence tautomerization. Chem 2019, 5, 108-121.

[3] Liu, C.; Sandoval-Salinas, M. E.; Hong, Y.; Gopalakrishna, T. Y.; Phan, H.; Aratani, N.; Herng, T. S.; Ding, J.; Yamada, H.; Kim, D.; Casanova, D.; Wu, J. Macrocyclic polyradicaloids with unusual superring structure and global aromaticity. Chem 2018, 4, 1586-1595.

[4] Gallagher, N.; Zhang, H.; Junghoefer, T.; Giangrisostomi, E.; Ovsyannikov, R.; Pink, M.; Rajca, S.; Casu, M. B.; Rajca, A. Thermally and magnetically robust triplet ground state diradical. Journal of the American Chemical Society 2019, 141, 4764-4774.

[5] Loboda, O.; Minaev, B.; Vahtras, O.; Schimmelpfennig, B.; Ågren, H.; Ruud, K.; Jonsson, D. Ab initio calculations of zero-field splitting parameters in linear polyacenes. Chemical Physics 2003, 286, 127-137.

[6] Richert, S.; Tait, C. E.; Timmel, C. R. Delocalisation of photoexcited triplet states probed by transient EPR and hyperfine spectroscopy. Journal of Magnetic Resonance 2017, 280, 103-116.

[7] Brandenburg, J. G.; Bannwarth, C.; Hansen, A.; Grimme, S. B97-3c: A revised low-cost variant of the B97-D density functional method. The Journal of Chemical Physics 2018, 148, 064104. 